\title{
Brønsted Acid-Catalyzed Allylboration: \\ Short and Stereodivergent Synthesis of All Four Eupomatilone Diastereomers with Crystallographic Assignments
}

\author{
Siu Hong Yu, Michael J. Ferguson, Robert McDonald, and Dennis G. Hall* \\ Department of Chemistry, University of Alberta, Edmonton, Alberta, Canada, T6G $2 G 2$
}

\section{Supporting Information}

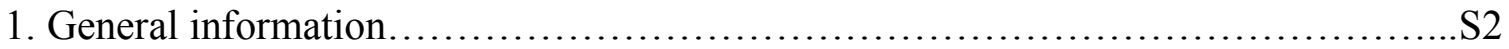

2. Preparation of 4,4-dimethyl-3-methylene-5-phenyl-dihydro-furan-2-one (3)

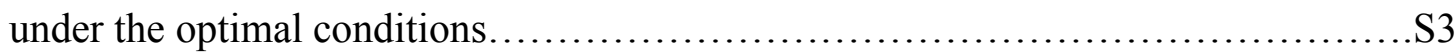

3. Preparation of methyl (2E)-2-[(4,4,5,5-tetramethyl-1,3,2-dioxaborolan-2-yl)methyl]-

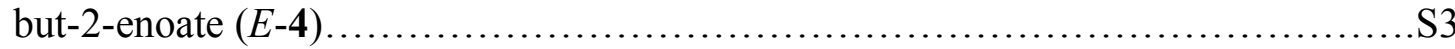

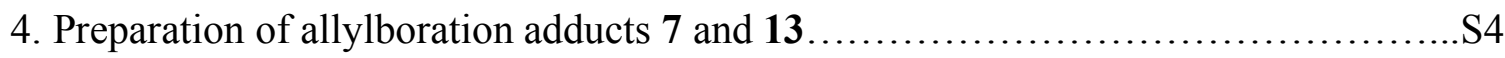

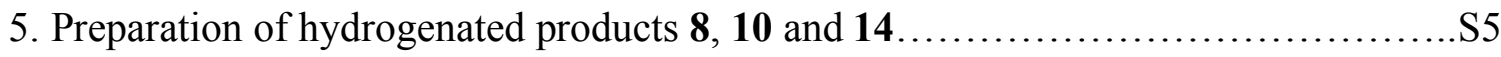

6. Preparation of Suzuki-coupling products 3,4-epi-1, 4-epi-1 and 3-epi-1..............S8

7. DBU-promoted epimerization of 3-epi-1 to eupomatilone-6 (1) ...................S11

8. Preliminary mechanistic investigations........................................... 11

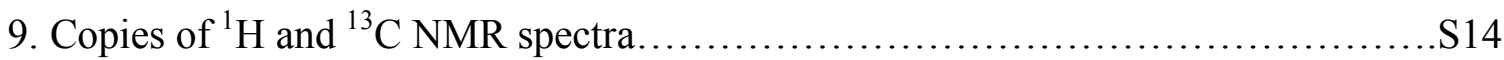

10. Summary table of ${ }^{1} \mathrm{H}$ NMR correspondences for the lactone moiety...............S24

11. ORTEP reproductions for $\mathbf{8}, \mathbf{1 0}, \mathbf{1 4}, 3,4$-epi-1, 4-epi-1........................S25

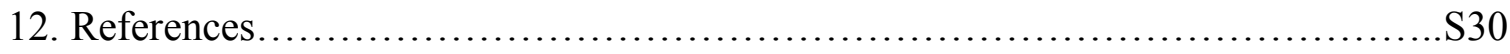




\section{General information}

Unless otherwise noted, all reactions were performed under an argon atmosphere using flame-dried glassware. Tetrahydrofuran was distilled over sodium-benzophenone ketyl. Toluene, $\mathrm{CH}_{2} \mathrm{Cl}_{2}$ and HMPA were distilled over $\mathrm{CaH}_{2}$ before use. $\mathrm{NH}_{4} \mathrm{Cl}_{(\mathrm{aq})}$ and $\mathrm{NaHCO}_{3(\mathrm{aq})}$ refer to saturated aqueous solutions. All liquid aldehydes were purified by Kugelrohr distillation prior to use. Methyllithium was titrated according to the Gilman double titration method. ${ }^{1}$ Iodomethaneboronate, ${ }^{2}$ chloromethaneboronate, ${ }^{10}$ and 2-bromo3,4,5-trimethoxybenzaldehyde ${ }^{3}$ were prepared according to literature procedures. TfOH was stored in a closed pear-shaped flask under Ar and placed in a jar filled with anhydrous calcium sulfate $\left(\right.$ Drierite $^{\circledR}$ ) which was then stored at $0{ }^{\circ} \mathrm{C}$. All other chemicals were used as received from commercial sources. Thin layer chromatography (TLC) was performed on Silica Gel $60 \mathrm{~F}_{254}$ plates and were visualized with UV light and aqueous $\mathrm{KMnO}_{4}$ solution. NMR spectra were recorded on 300,400 or $500 \mathrm{MHz}$ instruments. The residual solvent protons $\left({ }^{1} \mathrm{H}\right)$ or the solvent carbons $\left({ }^{13} \mathrm{C}\right)$ were used as internal standards. ${ }^{1} \mathrm{H}$ NMR data are presented as follows: chemical shift in ppm downfield from tetramethylsilane (multiplicity, integration, coupling constant). The following abbreviations are used in reporting NMR data: s, singlet; $d$, doublet; $t$, triplet; q, quartet; $\mathrm{dd}$, doublet of doublets; dq, doublet of quartets; dt, doublet of triplets; qt, quartet of triplets; m, multiplet. High resolution mass spectra were recorded by the University of Alberta Mass Spectrometry Services Laboratory using electron impact (EI) or electrospray (ES) ionization techniques. Elemental analyses were performed by the University of Alberta Micro-Analytical Lab. Infrared spectra and X-ray diffraction data were collected by the University of Alberta Spectral Services and X-Ray Crystallography Laboratory, respectively. 


\section{Preparation of 4,4-dimethyl-3-methylene-5-phenyl-dihydro-furan-2-one (3) under the optimal conditions}

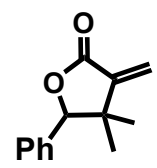

A solution of allylboronate $2^{4}(\mathrm{R}=(-)$-menthol, $71.1 \mathrm{mg}, 0.188 \mathrm{mmol})$ and benzaldehyde $(38 \mu \mathrm{L}, 0.37 \mathrm{mmol})$ in toluene $(0.2 \mathrm{~mL})$ was treated with $\mathrm{TfOH}(1.7 \mu \mathrm{L}$, $0.019 \mathrm{mmol}$ ) and stirred at $0{ }^{\circ} \mathrm{C}$ under $\mathrm{Ar}$ atmosphere for $16 \mathrm{~h}$. The mixture was then diluted with $\mathrm{NH}_{4} \mathrm{Cl}_{(\mathrm{aq})} / \mathrm{NH}_{4} \mathrm{OH}(9: 1 \mathrm{v} / \mathrm{v}, 3 \mathrm{~mL})$ and extracted with $\mathrm{Et}_{2} \mathrm{O}(3 \times 3 \mathrm{~mL})$. The combined extracts were washed with brine $(2 \times 3 \mathrm{~mL})$, dried with anhydrous $\mathrm{Na}_{2} \mathrm{SO}_{4}$ and concentrated. Flash chromatography $\left(15 \% \mathrm{Et}_{2} \mathrm{O} /\right.$ hexane) gave the lactone product 3 (38 $\mathrm{mg}, 0.19 \mathrm{mmol}, 99 \%$ ). This material possessed identical spectroscopic characteristics to those reported in the literature. ${ }^{4}$

\section{Preparation of methyl $(2 E)-2-[(4,4,5,5$-tetramethyl-1,3,2-dioxaborolan-2- yl)methyl]-but-2-enoate $(E-4)$}

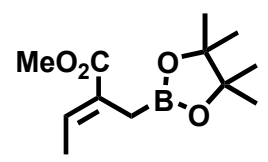

A slurry of recrystallized $\mathrm{CuBr} \cdot \mathrm{SMe}_{2}(833 \mathrm{mg}, 4.05 \mathrm{mmol})$ in anhydrous THF (11 $\mathrm{mL}$ ) at $0{ }^{\circ} \mathrm{C}$ under $\mathrm{Ar}$ atmosphere was treated with $\mathrm{MeLi}\left(1.60 \mathrm{M}\right.$ in $\mathrm{Et}_{2} \mathrm{O}, 5.1 \mathrm{~mL}, 8.1$ mmol) using an air-tight syringe. An orange precipitate was formed initially then disappeared as the addition proceeded. The resulting clear colourless solution was stirred at $0{ }^{\circ} \mathrm{C}$ for an additional $10 \mathrm{~min}$ and then cooled to $-78{ }^{\circ} \mathrm{C}$ in an acetone $/ \mathrm{CO}_{2}$ bath. $\mathrm{A}-78$ ${ }^{\circ} \mathrm{C}$ solution of methyl propiolate $(0.36 \mathrm{~mL}, 4.0 \mathrm{mmol})$ in $\mathrm{THF}(1 \mathrm{~mL})$ was added via cannulation with THF rinse $(3 \times 1 \mathrm{~mL})$. The resulting light brown mixture was stirred at $78{ }^{\circ} \mathrm{C}$ for $1 \mathrm{~h}$ and then injected with a $-78{ }^{\circ} \mathrm{C}$ solution of freshly distilled

iodomethaneboronate $^{2}(2.99 \mathrm{~g}, 11.2 \mathrm{mmol})$ in THF $(2 \mathrm{~mL})$ via cannulation with THF rinse $(3 \times 1 \mathrm{~mL})$. HMPA $(6.35 \mathrm{~mL}, 36.4 \mathrm{mmol})$ was quickly added. Stirring continued at $-78{ }^{\circ} \mathrm{C}$ for another $10 \mathrm{~min}$. The mixture was then brought to $0{ }^{\circ} \mathrm{C}$, stirred for $1 \mathrm{~h}$ and 
finally brought to rt, stirred for $1 \mathrm{~h}$. The milky slurry was quenched with $\mathrm{NH}_{4} \mathrm{Cl}_{(\mathrm{aq})}(20$ $\mathrm{mL})$. The layers were separated and the aqueous layer was extracted with $\mathrm{Et}_{2} \mathrm{O}(4 \times 20$ $\mathrm{mL})$. The combined organic layers were washed with water $(6 \times 20 \mathrm{~mL})$ and brine $(1 \times$ $20 \mathrm{~mL}$ ), dried with anhydrous $\mathrm{Na}_{2} \mathrm{SO}_{4}$, filtered and concentrated. Flash chromatography (25\% $\mathrm{Et}_{2} \mathrm{O} /$ hexane) gave the product as a yellowish liquid (740 $\mathrm{mg}, 3.12 \mathrm{mmol}, 77 \%$ ). This material possessed identical spectroscopic characteristics to those reported in the literature. $^{4}$

\section{Preparation of allylboration adducts 7 and $\mathbf{1 3}$}

rac-(4R*, 5S*)-5-(2-Bromo-3,4,5-trimethoxyphenyl)-4-methyl-3-methylene-dihydrofuran-2-one (7)

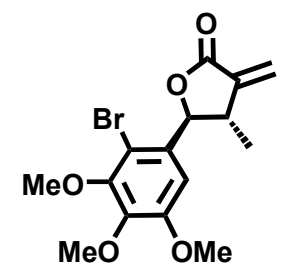

A slurry of allylboronate $E-4(323 \mathrm{mg}, 1.34 \mathrm{mmol})$ and 2-bromo-3,4,5trimethoxybenzaldehyde $6^{3}(740 \mathrm{mg}, 2.69 \mathrm{mmol})$ in toluene $(1.34 \mathrm{~mL})$ at $0{ }^{\circ} \mathrm{C}$ was treated with TfOH $(24 \mu \mathrm{L}, 0.27 \mathrm{mmol})$ and stirred at $0{ }^{\circ} \mathrm{C}$ under Ar atmosphere for $16 \mathrm{~h}$. The mixture was then diluted with $\mathrm{NH}_{4} \mathrm{Cl}_{(\mathrm{aq})} / \mathrm{NH}_{4} \mathrm{OH}(9: 1 \mathrm{v} / \mathrm{v}, 20 \mathrm{~mL})$ and extracted with $\mathrm{Et}_{2} \mathrm{O}(3 \times 20 \mathrm{~mL})$. The combined extracts were washed with brine $(2 \times 20 \mathrm{~mL})$, dried with anhydrous $\mathrm{Na}_{2} \mathrm{SO}_{4}$ and concentrated. Flash chromatography (25\% EtOAc/hexane) gave the lactone product 7 (272 $\mathrm{mg}, 0.762 \mathrm{mmol}, 57 \%)$.

${ }^{1} \mathrm{H} \mathrm{NMR}\left(500 \mathrm{MHz}, \mathrm{CDCl}_{3}\right): \delta 6.63(\mathrm{~s}, 1 \mathrm{H}), 6.32(\mathrm{~d}, 1 \mathrm{H}, J=2.7 \mathrm{~Hz}), 5.62(\mathrm{~d}, 1 \mathrm{H}$, $J=2.4 \mathrm{~Hz}$ ), $5.44(\mathrm{~d}, 1 \mathrm{H}, J=5.3 \mathrm{~Hz}), 3.91(\mathrm{~s}, 3 \mathrm{H}), 3.89$ (s, 3H), 3.84 (s, 3H), 2.98 (m, $1 \mathrm{H}), 1.46(\mathrm{~d}, 3 \mathrm{H}, J=7.0) ;{ }^{13} \mathrm{C}$ NMR $\left(100 \mathrm{MHz}, \mathrm{CDCl}_{3}\right): \delta 170.2,153.3,151.0,143.1$, 139.8, 134.0, 129.0, 122.1, 108.2, 105.4, 83.9, 61.0, 56.2, 43.5, 18.6; IR $\left(\mathrm{CH}_{2} \mathrm{Cl}_{2}\right.$ cast film, $\left.\mathrm{cm}^{-1}\right): 2969,2938,1769,1485,1396,1325,1248,1107,1000$; HRMS (EI, m/z) Calcd for $\mathrm{C}_{15} \mathrm{H}_{17}{ }^{81} \mathrm{BrO}_{5}: 358.02390$. Found: 358.02432 . 


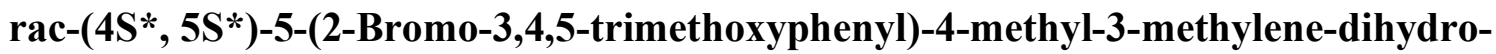
furan-2-one (13)

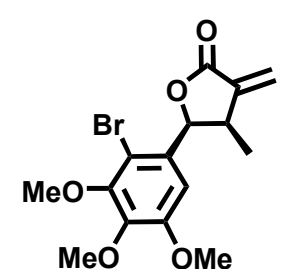

A slurry of allylboronate $E-4(358 \mathrm{mg}, 1.49 \mathrm{mmol})$ and 2-bromo-3,4,5trimethoxybenzaldehyde $6^{3}(410 \mathrm{mg}, 1.49 \mathrm{mmol})$ in toluene $(1.5 \mathrm{~mL})$ was heated at 110 ${ }^{\circ} \mathrm{C}$ under Ar atmosphere for $48 \mathrm{~h}$. A spatula tip of $p \mathrm{TSA} \cdot \mathrm{H}_{2} \mathrm{O}$ was then added and the mixture was stirred for $3 \mathrm{~h}$ at $\mathrm{rt}$. The reaction was quenched with $\mathrm{NaHCO}_{3(\mathrm{aq})}(20 \mathrm{~mL})$ and extracted with $\mathrm{Et}_{2} \mathrm{O}(3 \times 20 \mathrm{~mL})$. The combined extracts were washed with brine $(20$ $\mathrm{mL}$ ), dried with anhydrous $\mathrm{Na}_{2} \mathrm{SO}_{4}$ and concentrated. Flash chromatography (35\% EtOAc/hexane) gave the lactone product $13(394 \mathrm{mg}, 1.10 \mathrm{mmol}, 74 \%)$.

${ }^{1} \mathrm{H}$ NMR $\left(300 \mathrm{MHz}, \mathrm{CDCl}_{3}\right): \delta 6.69(\mathrm{~s}, 1 \mathrm{H}), 6.32(\mathrm{~d}, 1 \mathrm{H}, J=2.3 \mathrm{~Hz}), 5.82(\mathrm{dd}$, $1 \mathrm{H}, J=0.6,7.3 \mathrm{~Hz}), 5.66(\mathrm{~d}, 1 \mathrm{H}, J=2.0 \mathrm{~Hz}), 3.89(\mathrm{~s}, 3 \mathrm{H}), 3.88$ (s, 3H), $3.83(\mathrm{~s}, 3 \mathrm{H})$, $3.68(\mathrm{~m}, 1 \mathrm{H}), 0.78(\mathrm{~d}, 3 \mathrm{H}, J=7.3) ;{ }^{13} \mathrm{C} \mathrm{NMR}\left(100 \mathrm{MHz}, \mathrm{CDCl}_{3}\right): \delta 169.8,152.9,150.6$, $142.6,140.3,131.2,122.7,107.3,105.8,80.7,60.9,56.1,37.1,16.6 ; \mathrm{IR}\left(\mathrm{CH}_{2} \mathrm{Cl}_{2}\right.$ cast film, $\left.\mathrm{cm}^{-1}\right):$ 2971, 2938, 1772, 1484, 1397, 1324, 1244, 1108, 1004; HRMS (EI, m/z) Calcd for $\mathrm{C}_{15} \mathrm{H}_{17}{ }^{79} \mathrm{BrO}_{5}$ : 356.02594. Found: 356.02523. Calcd for $\mathrm{C}_{15} \mathrm{H}_{17}{ }^{81} \mathrm{BrO}_{5}$ : 358.0290. Found: 358.02347.

\section{Preparation of hydrogenated products 8,10 and 14}

rac-(3S*, 4S*, 5S*)-5-(2-Bromo-3,4,5-trimethoxyphenyl)-3,4-dimethyl-dihydrofuran-2-one (8)

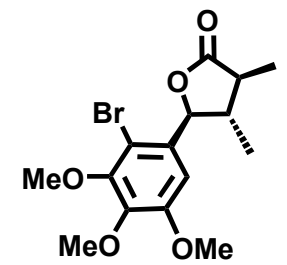


A solution of lactone $7(78 \mathrm{mg}, 0.22 \mathrm{mmol})$ in EtOH $(2.4 \mathrm{~mL})$ was hydrogenated at $1 \mathrm{~atm}$ with $10 \% \mathrm{Pd} / \mathrm{C}(7 \mathrm{mg})$ for $6 \mathrm{~h}$. The mixture was then filtered through Celite ${ }^{\circledR}$ 545 with EtOH rinse followed by solvent removal to give hydrogenated product 8 as the major diastereomer $(6: 1 \mathrm{dr})$. Flash chromatography (35\% EtOAc/hexane) separated the diastereomers and yielded the pure 8 (49 mg, $0.14 \mathrm{mmol}, 64 \%$,). Single crystals suitable for X-ray diffraction were obtained by dissolving $\mathbf{8}$ in hot pentane followed by storing the resulting solution at $0{ }^{\circ} \mathrm{C}$ in a closed vial.

${ }^{1} \mathrm{H}$ NMR $\left(300 \mathrm{MHz}, \mathrm{CDCl}_{3}\right): \delta 6.70(\mathrm{~s}, 1 \mathrm{H}), 5.44(\mathrm{~d}, 1 \mathrm{H}, J=9.5 \mathrm{~Hz}), 3.91(\mathrm{~s}$, 3H), $3.90(\mathrm{~s}, 3 \mathrm{H}), 3.87$ (s, 3H), $2.40(\mathrm{dq}, 1 \mathrm{H}, J=11.5,7.0 \mathrm{~Hz}), 2.01(\mathrm{~m}, 1 \mathrm{H}), 1.31$ (d, 3H, $J=7.0 \mathrm{~Hz}), 1.23(\mathrm{~d}, 3 \mathrm{H}, J=6.6 \mathrm{~Hz}) ;{ }^{13} \mathrm{C} \mathrm{NMR}\left(125 \mathrm{MHz}, \mathrm{CDCl}_{3}\right): \delta 178.4,153.3$, $150.6,143.4,132.5,109.6,106.1,83.9,61.0,60.9,56.2,48.6,43.3,14.9$, 12.9; IR $\left(\mathrm{CH}_{2} \mathrm{Cl}_{2}\right.$ cast film, $\left.\mathrm{cm}^{-1}\right): 2968,2937,1776,1486,1397,1333,1241,1169,1107,1002$; HRMS (EI, m/z) Calcd for $\mathrm{C}_{15} \mathrm{H}_{19}{ }^{79} \mathrm{BrO}_{5}$ : 358.04160. Found: 358.04184. Calcd for $\mathrm{C}_{15} \mathrm{H}_{19}{ }^{81} \mathrm{BrO}_{5}$ : 360.03955. Found: 360.03992; Anal. Calcd for $\mathrm{C}_{15} \mathrm{H}_{19} \mathrm{BrO}_{5}$ : C, 50.16; $\mathrm{H}$, 5.33. Found: C, 50.17; H, 5.35. X-ray crystallographic data can be found in the on-line CIF files.

rac-(3R*, 4S*, 5S*)-5-(2-Bromo-3,4,5-trimethoxyphenyl)-3,4-dimethyl-dihydrofuran-2-one (10)

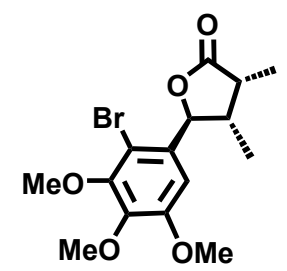

A solution of lactone $7(86 \mathrm{mg}, 0.24 \mathrm{mmol})$ in toluene $(12.5 \mathrm{~mL})$ was treated with Wilkinson's catalyst (56 mg, $0.060 \mathrm{mmol}$ ) and hydrogenated at $1 \mathrm{~atm}$ for $6 \mathrm{~h}$. Solvent removal gave the hydrogenated product $\mathbf{1 0}$ as the major diastereomer $(5: 1 \mathrm{dr})$. Flash chromatography (35\% EtOAc/hexane) separated the diastereomers and yielded the pure 10 (80 mg, $0.22 \mathrm{mmol}, 92 \%$,). Single crystals suitable for X-ray diffraction were obtained by dissolving $\mathbf{1 0}$ in hot pentane followed by storing the resulting solution at 0 ${ }^{\circ} \mathrm{C}$ in a closed vial. 
${ }^{1} \mathrm{H}$ NMR $\left(300 \mathrm{MHz}, \mathrm{CDCl}_{3}\right): \delta 6.63(\mathrm{~s}, 1 \mathrm{H}), 5.34(\mathrm{~d}, 1 \mathrm{H}, J=2.5 \mathrm{~Hz}), 3.91(\mathrm{~s}$, $3 \mathrm{H}), 3.88(\mathrm{~s}, 3 \mathrm{H}), 3.85(\mathrm{~s}, 3 \mathrm{H}), 2.74$ (app quintet, $1 \mathrm{H}, J=7.5 \mathrm{~Hz}), 2.58(\mathrm{~m}, 1 \mathrm{H}), 1.24$ (d, $3 \mathrm{H}, J=7.2 \mathrm{~Hz}), 1.19$ (d, 3H, $J=7.3 \mathrm{~Hz}) ;{ }^{13} \mathrm{C}$ NMR $\left(125 \mathrm{MHz}, \mathrm{CDCl}_{3}\right): \delta$ 179.7, 153.1, $151.2,142.7,133.8,107.4,104.7,84.2,61.0,56.2,41.1,36.3,14.2,9.5 ; \mathrm{IR}\left(\mathrm{CH}_{2} \mathrm{Cl}_{2}\right.$ cast film, $\mathrm{cm}^{-1}$ ): 2973, 2939, 1779, 1483, 1396, 1330, 1240, 1168, 1110, 1000; HRMS (EI, $\mathrm{m} / \mathrm{z}$ ) Calcd for $\mathrm{C}_{15} \mathrm{H}_{19}{ }^{79} \mathrm{BrO}_{5}$ : 358.04160. Found: 358.04156. Calcd for $\mathrm{C}_{15} \mathrm{H}_{19}{ }^{81} \mathrm{BrO}_{5}$ : 360.03955. Found: 360.04031; Anal. Calcd for $\mathrm{C}_{15} \mathrm{H}_{19} \mathrm{BrO}_{5}$ : C, 50.16; H, 5.33. Found: $\mathrm{C}$, 49.91; H, 5.32. X-ray crystallographic data can be found in the on-line CIF files.

rac-(3S*, 4R*, 5S*)-5-(2-Bromo-3,4,5-trimethoxyphenyl)-3,4-dimethyl-dihydrofuran-2-one (14)

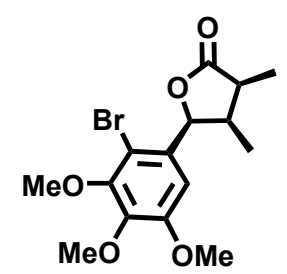

A solution of lactone $\mathbf{1 3}(55 \mathrm{mg}, 0.16 \mathrm{mmol})$ in toluene $(8 \mathrm{~mL})$ was treated with Wilkinson's catalyst (36 mg, $0.039 \mathrm{mmol}$ ) and hydrogenated at $1 \mathrm{~atm}$ for $6 \mathrm{~h}$. Solvent removal followed by flash chromatography ( $35 \%$ EtOAc/hexane) gave the hydrogenated product 14 as the only diastereomer observed (44 $\mathrm{mg}, 0.13 \mathrm{mmol}, 79 \%$ ). Single crystals suitable for X-ray diffraction were obtained by dissolving $\mathbf{1 4}$ in hot pentane followed by storing the resulting solution at $0{ }^{\circ} \mathrm{C}$ in a closed vial.

${ }^{1} \mathrm{H}$ NMR (300 MHz, $\left.\mathrm{CDCl}_{3}\right): \delta 6.81(\mathrm{~s}, 1 \mathrm{H}), 5.63(\mathrm{dd}, 1 \mathrm{H}, J=0.6,5.0 \mathrm{~Hz}), 3.90$ (s, 3H), $3.88(\mathrm{~s}, 3 \mathrm{H}), 3.85(\mathrm{~s}, 3 \mathrm{H}), 3.21(\mathrm{~m}, 1 \mathrm{H}), 3.03$ (app quintet, $1 \mathrm{H}, J=7.2 \mathrm{~Hz}), 1.22$ (d, $3 \mathrm{H}, J=7.2 \mathrm{~Hz}), 0.52$ (d, 3H, $J=7.3 \mathrm{~Hz}) ;{ }^{13} \mathrm{C} \mathrm{NMR}\left(125 \mathrm{MHz}, \mathrm{CDCl}_{3}\right): \delta 178.5$, 153.0, 150.8, 142.5, 131.1, 106.6, 106.3, 81.9, 61.1, 61.0, 56.3, 40.5, 37.3, 10.0, 9.7; IR $\left(\mathrm{CH}_{2} \mathrm{Cl}_{2}\right.$ cast film, $\left.\mathrm{cm}^{-1}\right): 2974,2939,1780,1484,1396,1338,1242,1170,1108,997$; HRMS (EI, m/z) Calcd for $\mathrm{C}_{15} \mathrm{H}_{19}{ }^{79} \mathrm{BrO}_{5}: 358.04160$. Found: 358.04174. Calcd for $\mathrm{C}_{15} \mathrm{H}_{19}{ }^{81} \mathrm{BrO}_{5}$ : 360.03955. Found: 360.03961; Anal. Calcd for $\mathrm{C}_{15} \mathrm{H}_{19} \mathrm{BrO}_{5}$ : C, 50.16; H, 5.33. Found: C, 49.63; H, 5.33. X-ray crystallographic data can be found in the on-line CIF files. 


\section{Preparation of Suzuki-coupling ${ }^{5}$ products 3,4-epi-1, 4-epi-1 and 3-epi-1}

\section{3,4-epi-1}

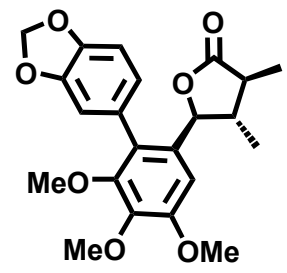

An oven-dried resealable Schlenk tube containing a magnetic stir bar was charged with arylbromide 8 (90 mg, $0.25 \mathrm{mmol})$, boronic acid 9 (62 mg, $0.38 \mathrm{mmol}), \mathrm{K}_{3} \mathrm{PO}_{4}(106$ $\mathrm{mg}, 0.5 \mathrm{mmol}), \mathrm{Pd}(\mathrm{OAc})_{2}(0.6 \mathrm{mg}, 0.0025 \mathrm{mmol})$ and SPhos ligand $(2.3 \mathrm{mg}, 0.005$ mmol). Capped with a rubber septum, the Schlenk tube was evacuated and backfilled with $\operatorname{Ar}$ (this sequence was repeated four times). Toluene $(0.5 \mathrm{~mL})$ was then added and the resulting mixture was degassed. Backfilled with Ar, the Schlenk tube was quickly sealed with a Teflon ${ }^{\circledR}$ screwcap. The mixture was heated at $110{ }^{\circ} \mathrm{C}$ with vigorous stirring for $12 \mathrm{~h}$. Cooled to rt, the mixture was diluted with $\mathrm{Et}_{2} \mathrm{O}$ followed by filtration through a thin pad of silica gel with $\mathrm{Et}_{2} \mathrm{O}$ rinse. Solvent evaporation followed by flash chromatography (40\% EtOAc/hexane) gave 3,4-epi-1 as the only diastereomer observed (96 mg, $0.24 \mathrm{mmol}, 98 \%$ ). Single crystals suitable for X-ray diffraction were obtained by dissolving 3,4-epi-1 in hot $\mathrm{Et}_{2} \mathrm{O}$ followed by storing the resulting solution at $\mathrm{rt}$ in a bigger, screwcapped vial containing hexane.

${ }^{1} \mathrm{H}$ NMR (500 MHz, $\mathrm{CDCl}_{3}$, two atropisomers): $\delta 6.85$ (s, 1H), $6.84(\mathrm{~s}, 1 \mathrm{H}), 6.72$ (d, 1H, $J=1.6 \mathrm{~Hz}), 6.71-6.68(\mathrm{~m}, 4 \mathrm{H}), 6.64(\mathrm{dd}, 1 \mathrm{H}, J=1.7,7.9 \mathrm{~Hz}), 6.00(\mathrm{~m}, 2 \mathrm{H}), 5.99$ $(\mathrm{m}, 2 \mathrm{H}), 4.80(\mathrm{~d}, 1 \mathrm{H}, J=9.8 \mathrm{~Hz}), 4.78(\mathrm{~d}, 1 \mathrm{H}, J=9.8 \mathrm{~Hz}), 3.90(\mathrm{~s}, 6 \mathrm{H}), 3.89(\mathrm{~s}, 6 \mathrm{H})$, $3.62(\mathrm{~s}, 3 \mathrm{H}), 3.61(\mathrm{~s}, 3 \mathrm{H}), 2.14(\mathrm{~m}, 2 \mathrm{H}), 2.03(\mathrm{~m}, 2 \mathrm{H}), 1.23(\mathrm{~d}, 6 \mathrm{H}, J=6.9 \mathrm{~Hz}), 0.87$ (d, $3 \mathrm{H}, J=6.4 \mathrm{~Hz}), 0.86(\mathrm{~d}, 3 \mathrm{H}, J=6.4 \mathrm{~Hz}) ;{ }^{13} \mathrm{C} \mathrm{NMR}\left(125 \mathrm{MHz}, \mathrm{CDCl}_{3}\right): \delta 178.5,178.4$, $153.2,151.2,151.1,147.4,147.3,146.8,142.5,130.7,130.6,129.8,128.9,128.8,124.2$, 123.3, 111.3, 110.5, 108.0, 107.8, 105.2, 105.1, 101.1, 101.0, 82.6, 82.5, 61.0, 60.9, 60.7, 56.1, 47.4, 43.2, 14.2, 12.8; IR $\left(\mathrm{CH}_{2} \mathrm{Cl}_{2}\right.$ cast film, $\left.\mathrm{cm}^{-1}\right): 2966,2935,1774,1484,1459$, 1324, 1238, 1039, 982; HRMS (EI, m/z) Calcd for $\mathrm{C}_{22} \mathrm{H}_{24} \mathrm{O}_{7}$ : 400.15219. Found: 400.15220. X-ray crystallographic data can be found in the on-line CIF files. The compound crystallized into a single atropisomer. 


\section{4-epi-1}

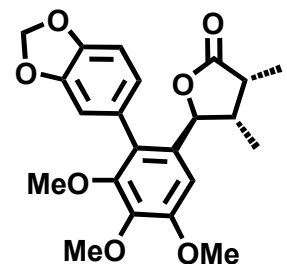

An oven-dried resealable Schlenk tube containing a magnetic stir bar was charged with arylbromide $10(90 \mathrm{mg}, 0.25 \mathrm{mmol})$, boronic acid 9 (62 mg, $0.38 \mathrm{mmol}), \mathrm{K}_{3} \mathrm{PO}_{4}$ (106 mg, $0.5 \mathrm{mmol}), \mathrm{Pd}(\mathrm{OAc})_{2}(0.6 \mathrm{mg}, 0.0025 \mathrm{mmol})$ and SPhos ligand (2.3 $\mathrm{mg}, 0.005$ mmol). Capped with a rubber septum, the Schlenk tube was evacuated and backfilled with $\operatorname{Ar}$ (this sequence was repeated four times). Toluene $(0.5 \mathrm{~mL})$ was then added and the resulting mixture was degassed. Backfilled with Ar, the Schlenk tube was quickly sealed with a Teflon ${ }^{\circledR}$ screwcap. The mixture was heated at $110{ }^{\circ} \mathrm{C}$ with vigorous stirring for $12 \mathrm{~h}$. Cooled to rt, the mixture was diluted with $\mathrm{Et}_{2} \mathrm{O}$ followed by filtration through a thin pad of silica gel with $\mathrm{Et}_{2} \mathrm{O}$ rinse. Solvent evaporation followed by flash chromatography (40\% EtOAc/hexane) gave 4-epi-1 along with ca. $10 \%$ of 3,4-epi-1 (88 $\mathrm{mg}, 0.22 \mathrm{mmol}, 88 \%$ total yield). The major diastereomer 4-epi-1 possessed identical spectroscopic characteristics to those reported in the literature. ${ }^{6}$ Single crystals suitable for X-ray diffraction were obtained by dissolving 4-epi-1 in hot $\mathrm{Et}_{2} \mathrm{O}$ followed by storing the resulting solution at $\mathrm{rt}$ in a bigger, screwcapped vial containing hexane.

${ }^{1} \mathrm{H}$ NMR (500 MHz, $\mathrm{CDCl}_{3}$, two atropisomers): $\delta 6.90$ (s, 1H), 6.88 (s, 1H), 6.75$6.61(\mathrm{~m}, 6 \mathrm{H}), 6.02(\mathrm{~m}, 4 \mathrm{H}), 5.11(\mathrm{~d}, 1 \mathrm{H}, J=4.5 \mathrm{~Hz}), 5.01(\mathrm{~d}, 1 \mathrm{H}, J=4.5 \mathrm{~Hz}), 3.90(\mathrm{~s}$, $6 \mathrm{H}), 3.88(\mathrm{~s}, 6 \mathrm{H}), 3.65(\mathrm{~s}, 3 \mathrm{H}), 3.64(\mathrm{~s}, 3 \mathrm{H}), 2.75(\mathrm{~m}, 2 \mathrm{H}), 2.39(\mathrm{~m}, 2 \mathrm{H}), 1.08(\mathrm{~d}, 3 \mathrm{H}, J=$ $7.3 \mathrm{~Hz}), 1.07(\mathrm{~d}, 3 \mathrm{H}, J=7.3 \mathrm{~Hz}), 0.69$ (d, 3H, $J=7.1 \mathrm{~Hz}), 0.66(\mathrm{~d}, 3 \mathrm{H}, J=7.1 \mathrm{~Hz}) ;{ }^{13} \mathrm{C}$ NMR (125 MHz, $\left.\mathrm{CDCl}_{3}\right)$ : $\delta$ 179.9, 153.1, 153.0, 151.8, 151.7, 147.7, 146.9, 146.8, 142.0, $132.8,129.0,128.9,124.1,122.9,111.2,110.1,108.4,108.2,103.6,101.2,101.1,82.8$, $61.1,61.0,60.8,56.2,41.6,41.5,37.1,13.3,9.8$. X-ray crystallographic data can be found in the on-line CIF files. The compound crystallized into a single atropisomer. 


\section{3-epi-1}

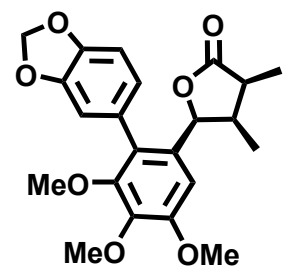

An oven-dried resealable Schlenk tube containing a magnetic stir bar was charged with arylbromide 14 (92 $\mathrm{mg}, 0.26 \mathrm{mmol})$, boronic acid 9 (63 mg, $0.38 \mathrm{mmol}), \mathrm{K}_{3} \mathrm{PO}_{4}$ (108 mg, $0.51 \mathrm{mmol}), \mathrm{Pd}(\mathrm{OAc})_{2}(2.8 \mathrm{mg}, 0.012 \mathrm{mmol})$ and SPhos ligand (12 mg, 0.026 mmol). Capped with a rubber septum, the Schlenk tube was evacuated and backfilled with $\operatorname{Ar}$ (this sequence was repeated four times). Toluene $(0.5 \mathrm{~mL})$ was then added and the resulting mixture was degassed. Backfilled with Ar, the Schlenk tube was quickly sealed with a Teflon ${ }^{\circledR}$ screwcap. The mixture was heated at $110{ }^{\circ} \mathrm{C}$ with vigorous stirring for $12 \mathrm{~h}$. Cooled to rt, the mixture was diluted with $\mathrm{Et}_{2} \mathrm{O}$ followed by filtration through a thin pad of silica gel with $\mathrm{Et}_{2} \mathrm{O}$ rinse. Solvent evaporation followed by flash chromatography (35\% EtOAc/hexane) gave 3-epi-1 along with ca. $17 \%$ of 1 (100 mg, $0.25 \mathrm{mmol}, 96 \%$ total yield). The major diastereomer 3-epi-1 possessed identical spectroscopic characteristics to those reported in the literature. ${ }^{7}$

${ }^{1} \mathrm{H}$ NMR (300 MHz, $\mathrm{CDCl}_{3}$, two atropisomers): $\delta 6.88(\mathrm{~s}, 1 \mathrm{H}), 6.86(\mathrm{~s}, 1 \mathrm{H}), 6.84$ $(\mathrm{s}, 1 \mathrm{H}), 6.82(\mathrm{~s}, 1 \mathrm{H}), 6.73(\mathrm{~d}, 1 \mathrm{H}, J=1.6 \mathrm{~Hz}), 6.70(\mathrm{dd}, 1 \mathrm{H}, J=1.6,7.8 \mathrm{~Hz}), 6.62(\mathrm{~d}, 1 \mathrm{H}$, $J=1.6 \mathrm{~Hz}), 6.58(\mathrm{dd}, 1 \mathrm{H}, J=1.7,7.9 \mathrm{~Hz}), 6.04(\mathrm{~m}, 2 \mathrm{H}), 6.02(\mathrm{~m}, 2 \mathrm{H}), 5.41$ (d, 1H, $J=$ $4.9 \mathrm{~Hz}), 5.32(\mathrm{~d}, 1 \mathrm{H}, J=4.9 \mathrm{~Hz}), 3.91(\mathrm{~s}, 12 \mathrm{H}), 3.66(\mathrm{~s}, 3 \mathrm{H}), 3.65(\mathrm{~s}, 3 \mathrm{H}), 2.75(\mathrm{~m}, 2 \mathrm{H})$, $2.20(\mathrm{~m}, 2 \mathrm{H}), 1.12(\mathrm{~d}, 6 \mathrm{H}, J=7.2 \mathrm{~Hz}), 0.56$ (app triplet, $6 \mathrm{H}, J=7.2 \mathrm{~Hz}) ;{ }^{13} \mathrm{C} \mathrm{NMR}(125$ $\left.\mathrm{MHz}, \mathrm{CDCl}_{3}\right): \delta 178.6,152.7,151.4,147.6,147.5,146.9,146.8,141.4,130.1,129.1$, $129.0,126.3,126.2,123.4,122.2,110.5,109.5,108.3,108.1,104.8,104.7,101.1,80.4$, $80.3,61.1,61.0,60.7,56.1,40.6,38.7,38.5,9.8,9.7,9.6$. 


\section{DBU-promoted epimerization ${ }^{8}$ of 3-epi-1 to eupomatilone-6 (1)}

In a resealable Schlenk tube, a small mixture which contained mainly 3-epi-1 in 1:1 toluene/MeOH ( $2 \mathrm{~mL})$ was treated with $\mathrm{DBU}$ ( $\sim 1.5$ equiv.). The Schlenk tube was sealed with a Teflon ${ }^{\circledR}$ screwcap and the mixture was heated at $80{ }^{\circ} \mathrm{C}$ with vigorous stirring for 2 days. The now almost 1:1 (based on ${ }^{1} \mathrm{H}$ NMR) mixture of 3-epi-1 and eupomatilone-6 (1) was submitted to preparative TLC (20\% EtOAc/hexane with multiple developments) ${ }^{7}$ and an analytically pure sample of 1 was obtained by deabsorption with $\mathrm{CH}_{2} \mathrm{Cl}_{2}$, filtration and concentration. This material possessed identical spectroscopic characteristics to those reported in the literature. ${ }^{7}$

${ }^{1} \mathrm{H}$ NMR (500 MHz, $\mathrm{CDCl}_{3}$, two atropisomers): $\delta 6.88(\mathrm{~d}, 1 \mathrm{H}, J=8.1 \mathrm{~Hz}), 6.87$ $(\mathrm{d}, 1 \mathrm{H}, J=8.1 \mathrm{~Hz}), 6.73(\mathrm{~d}, 1 \mathrm{H}, J=1.4 \mathrm{~Hz}), 6.70(\mathrm{dd}, 1 \mathrm{H}, J=1.6,7.8 \mathrm{~Hz}), 6.67(\mathrm{~s}, 1 \mathrm{H})$, $6.66(\mathrm{~s}, 1 \mathrm{H}), 6.65(\mathrm{~d}, 1 \mathrm{H}, J=1.4 \mathrm{~Hz}), 6.59(\mathrm{dd}, 1 \mathrm{H}, J=1.6,8.1 \mathrm{~Hz}), 6.03(\mathrm{~m}, 2 \mathrm{H}), 6.02$ (m, 2H), 5.65 (d, 1H, $J=7.0 \mathrm{~Hz}), 5.53(\mathrm{~d}, 1 \mathrm{H}, J=7.0 \mathrm{~Hz}), 3.91(\mathrm{~s}, 6 \mathrm{H}), 3.89(\mathrm{~s}, 6 \mathrm{H})$, $3.65(\mathrm{~s}, 3 \mathrm{H}), 3.64(\mathrm{~s}, 3 \mathrm{H}), 2.36(\mathrm{~m}, 2 \mathrm{H}), 2.05-1.96(\mathrm{~m}, 2 \mathrm{H}), 1.20(\mathrm{~d}, 3 \mathrm{H}, J=7.6 \mathrm{~Hz}), 1.19$ $(\mathrm{d}, 3 \mathrm{H}, J=7.6 \mathrm{~Hz}), 0.73(\mathrm{~d}, 3 \mathrm{H}, J=7.2 \mathrm{~Hz}), 0.70(\mathrm{~d}, 3 \mathrm{H}, J=7.2 \mathrm{~Hz})$.

\section{Preliminary mechanistic investigations}

\section{Treatment of allylboronate $E-4$ with $\mathrm{TfOH}$}

Isomerically pure allylboronate $E-4(23 \mathrm{mg}, 0.096 \mathrm{mmol})$ was dissolved in toluene $(0.1 \mathrm{~mL})$, cooled to $0{ }^{\circ} \mathrm{C}$, treated with $\mathrm{TfOH}(1.7 \mu \mathrm{L}, 0.019 \mathrm{mmol})$ and stirred at $0{ }^{\circ} \mathrm{C}$ under Ar atmosphere for $16 \mathrm{~h}$. The mixture was then diluted with $\mathrm{NH}_{4} \mathrm{Cl}_{(\mathrm{aq})} / \mathrm{NH}_{4} \mathrm{OH}$ $(9: 1 \mathrm{v} / \mathrm{v}, 2 \mathrm{~mL})$ and extracted with $\mathrm{Et}_{2} \mathrm{O}(3 \times 2 \mathrm{~mL})$. The combined extracts were washed with brine $(1 \times 2 \mathrm{~mL})$, dried with anhydrous $\mathrm{Na}_{2} \mathrm{SO}_{4}$, filtered and concentrated. ${ }^{1} \mathrm{H} \mathrm{NMR}$ analysis of the crude reaction mixture showed mainly starting allylboronate $E-4$ (ca. 66 \%) along with other unidentified side products. No Z-4 isomer was observed. 


\section{Treatment of lactone 13 with TfOH}

Diastereomerically pure lactone 13 (34 $\mathrm{mg}, 0.095 \mathrm{mmol})$ was dissolved in toluene $(0.1 \mathrm{~mL})$, cooled to $0{ }^{\circ} \mathrm{C}$, treated with $\mathrm{TfOH}(1.7 \mu \mathrm{L}, 0.019 \mathrm{mmol})$ and stirred at $0{ }^{\circ} \mathrm{C}$ under $\mathrm{Ar}$ atmosphere for $16 \mathrm{~h}$. The mixture was then diluted with $\mathrm{NH}_{4} \mathrm{Cl}_{(\mathrm{aq})} / \mathrm{NH}_{4} \mathrm{OH}(9: 1$ $\mathrm{v} / \mathrm{v}, 2 \mathrm{~mL})$ and extracted with $\mathrm{Et}_{2} \mathrm{O}(3 \times 2 \mathrm{~mL})$. The combined extracts were washed with brine $(1 \times 2 \mathrm{~mL})$, dried with anhydrous $\mathrm{Na}_{2} \mathrm{SO}_{4}$, filtered and concentrated. ${ }^{1} \mathrm{H} \mathrm{NMR}$ analysis of the crude reaction mixture showed mainly starting lactone $\mathbf{1 3}$ (ca. $80 \%$ ) along with ca. $20 \%$ of lactone 7 .

\section{Preparation of ethyl (2Z)-2-[(4,4,5,5-tetramethyl-1,3,2-dioxaborolan-2-yl)methyl] but-2-enoate $(Z-4)^{9}$}

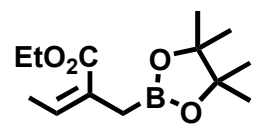

To a stirring solution of HMPA $(1.56 \mathrm{~mL}, 8.97 \mathrm{mmol})$ in toluene $(9 \mathrm{~mL})$ under $\mathrm{Ar}$ atmosphere at $0{ }^{\circ} \mathrm{C}$, DIBALH $(1.0 \mathrm{M}$ in toluene, $4.48 \mathrm{~mL}, 4.48 \mathrm{mmol})$ was added dropwise. After stirring at $0{ }^{\circ} \mathrm{C}$ for $1 \mathrm{~h}$, freshly distilled ethyl 2-butynoate $(0.35 \mathrm{~mL}, 3.0$ mmol) was added and stirring continued at $0{ }^{\circ} \mathrm{C}$ for another $5 \mathrm{~h}$. Freshly distilled chloromethaneboronate $^{10}(632 \mathrm{mg}, 3.58 \mathrm{mmol})$ in toluene $(2 \mathrm{~mL})$ was added via cannulation with toluene rinse $(3 \times 1 \mathrm{~mL})$ and the resulting mixture was allowed to warm up to $\mathrm{rt}$ and stirred under Ar atmosphere for $16 \mathrm{~h}$. The reaction was then quenched with 1 $\mathrm{M} \mathrm{HCl}(16 \mathrm{~mL})$ and extracted with $\mathrm{Et}_{2} \mathrm{O}(4 \times 20 \mathrm{~mL})$. The combined organic phase was washed with $1 \mathrm{M} \mathrm{HCl}(3 \times 16 \mathrm{~mL}), \mathrm{NaHCO}_{3(\mathrm{aq})}(1 \times 16 \mathrm{~mL})$, water $(1 \times 16 \mathrm{~mL})$, brine $(1$ $\times 16 \mathrm{~mL}$ ), dried with anhydrous $\mathrm{Na}_{2} \mathrm{SO}_{4}$, filtered and concentrated. Flash chromatography ( $25 \% \mathrm{Et}_{2} \mathrm{O} /$ hexane) gave the product $Z-4$ along with ca. $20 \%$ of the $E$ isomer (224 mg, $0.882 \mathrm{mmol}, 30 \%$ combined yield).

${ }^{1} \mathrm{H}$ NMR (300 MHz, $\mathrm{CDCl}_{3}$ ): $\delta 6.04$ (qt, 1H, $\left.J=7.2,1.3 \mathrm{~Hz}\right), 4.20$ (q, 2H, $J=7.1$ $\mathrm{Hz}), 2.00(\mathrm{dt}, 3 \mathrm{H}, J=7.2,1.3 \mathrm{~Hz}), 1.84(\mathrm{~s}, 2 \mathrm{H}), 1.30$ (t, 3H, $J=7.1 \mathrm{~Hz}), 1.24(\mathrm{~s}, 12 \mathrm{H})$; ${ }^{13} \mathrm{C}$ NMR $\left(125 \mathrm{MHz}, \mathrm{CDCl}_{3}\right): \delta 168.0,136.8,129.1,83.2,60.0,24.7,15.8,14.2$; IR (microscope, $\mathrm{cm}^{-1}$ ): 2980, 1717, 1353, 1323, 1146, 968, 847; HRMS (EI, m/z) Calcd for $\mathrm{C}_{13} \mathrm{H}_{23}{ }^{11} \mathrm{BO}_{4}$ : 254.16985. Found: 254.16918. 
Preparation of rac-(4R*, 5S*)-5-(2-Bromo-3,4,5-trimethoxyphenyl)-4-methyl-3methylene-dihydro-furan-2-one (7) by TfOH-catalyzed allylboration with $Z$-4

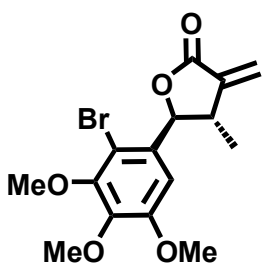

A slurry of Z-4 $(Z / E=80: 20,60.4 \mathrm{mg}, 0.238 \mathrm{mmol})$ and 2-bromo-3,4,5trimethoxybenzaldehyde $6^{3}(131 \mathrm{mg}, 0.476 \mathrm{mmol})$ in toluene $(0.24 \mathrm{~mL})$ at $0{ }^{\circ} \mathrm{C}$ was treated with $\mathrm{TfOH}(4.2 \mu \mathrm{L}, 0.048 \mathrm{mmol})$ and stirred at $0{ }^{\circ} \mathrm{C}$ under $\mathrm{Ar}$ atmosphere for 16 h. The mixture was then diluted with $\mathrm{NH}_{4} \mathrm{Cl}_{(\mathrm{aq})} / \mathrm{NH}_{4} \mathrm{OH}(9: 1 \mathrm{v} / \mathrm{v}, 4 \mathrm{~mL})$ and extracted with $\mathrm{Et}_{2} \mathrm{O}(3 \times 4 \mathrm{~mL})$. The combined extracts were washed with brine $(2 \times 2 \mathrm{~mL})$, dried with anhydrous $\mathrm{Na}_{2} \mathrm{SO}_{4}$, filtered and concentrated. Flash chromatography (25\% EtOAc/hexane) gave the diastereomerically pure lactone product 7 (85 $\mathrm{mg}, 0.238 \mathrm{mmol}$, $100 \%$ ). This material possessed identical spectroscopic characteristics to those reported above for 7 . 


\section{Copies of ${ }^{1} \mathrm{H}$ and ${ }^{13} \mathrm{C}$ NMR spectra}

$\left(4 \mathrm{R}^{*}, 5 \mathrm{~S}^{*}\right)-5$-(2-Bromo-3,4,5-trimethoxyphenyl)-4-methyl-3-methylene-dihydro-furan-2-one (7) ${ }^{1} \mathrm{H}(500 \mathrm{MHz})$ and ${ }^{13} \mathrm{C}(100 \mathrm{MHz}) \mathrm{NMR}$ in $\mathrm{CDCl}_{3}$
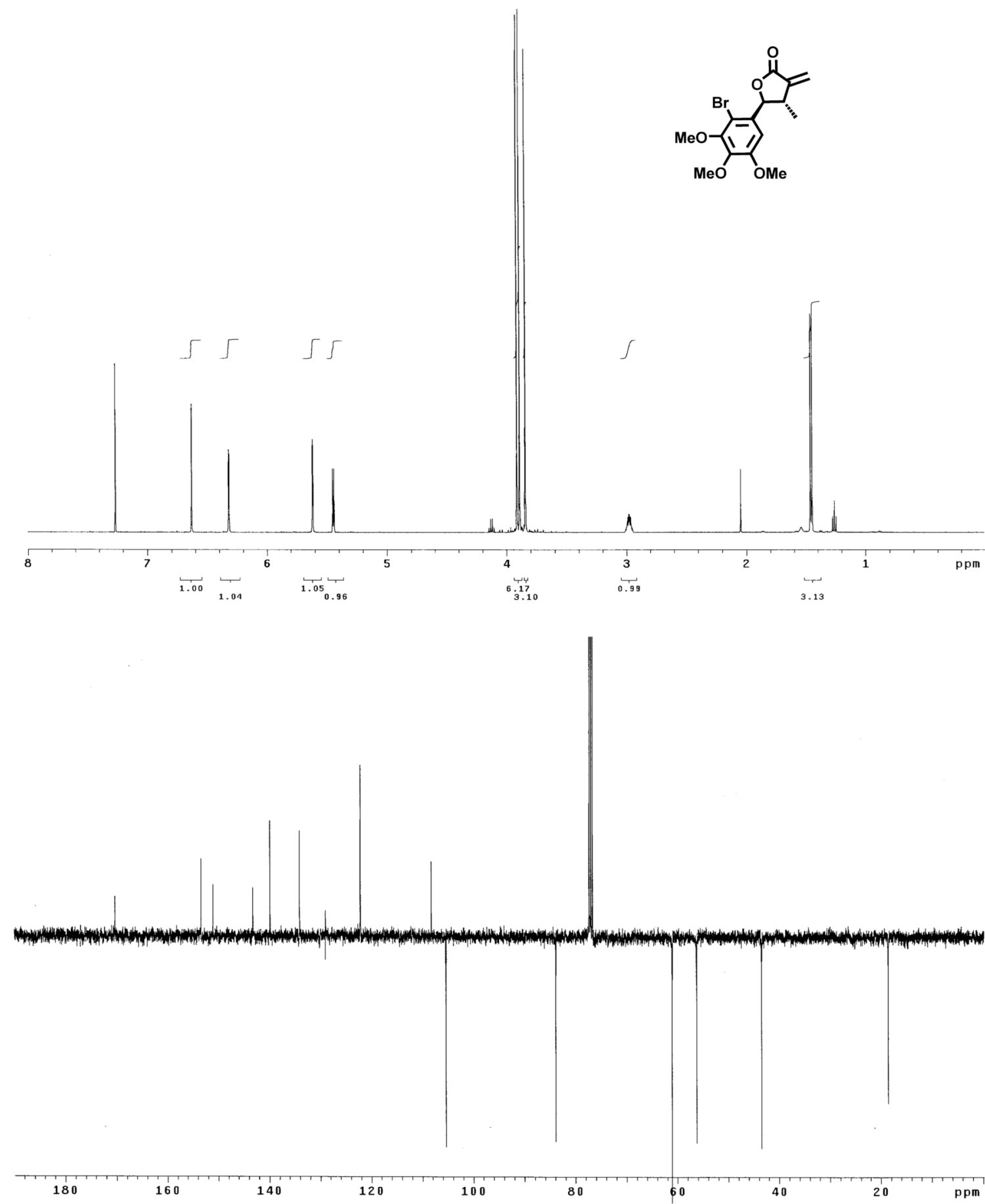


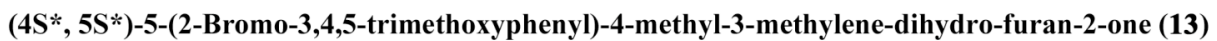
${ }^{1} \mathrm{H}(300 \mathrm{MHz})$ and ${ }^{13} \mathrm{C}(100 \mathrm{MHz}) \mathrm{NMR}$ in $\mathrm{CDCl}_{3}$
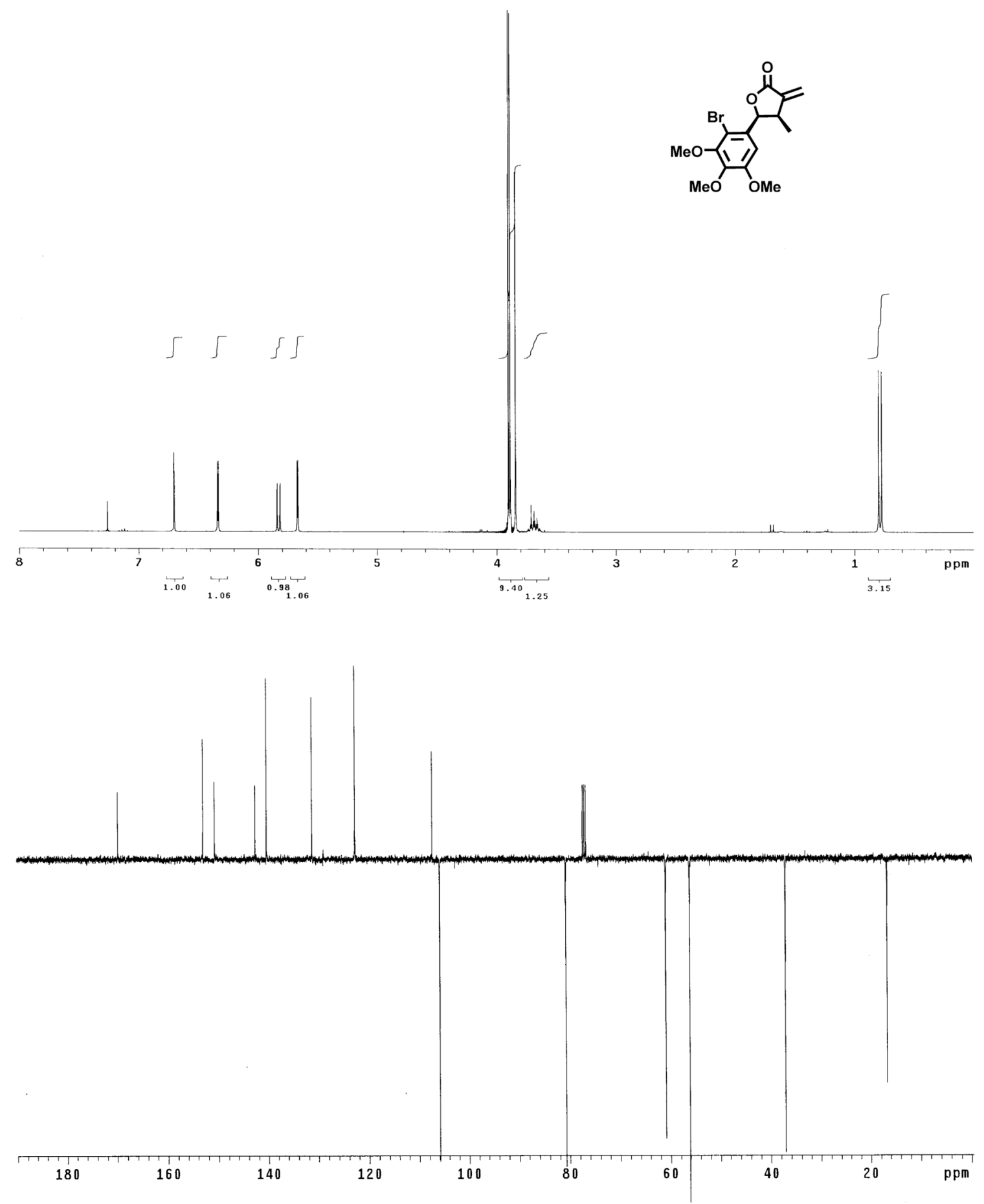
$\left(3 \mathrm{~S}^{*}, 4 \mathrm{~S}^{*}, 5 \mathrm{~S}^{*}\right)$-5-(2-Bromo-3,4,5-trimethoxyphenyl)-3,4-dimethyl-dihydro-furan-2-one (8) ${ }^{1} \mathrm{H}(300 \mathrm{MHz})$ and ${ }^{13} \mathrm{C}(125 \mathrm{MHz}) \mathrm{NMR}$ in $\mathrm{CDCl}_{3}$
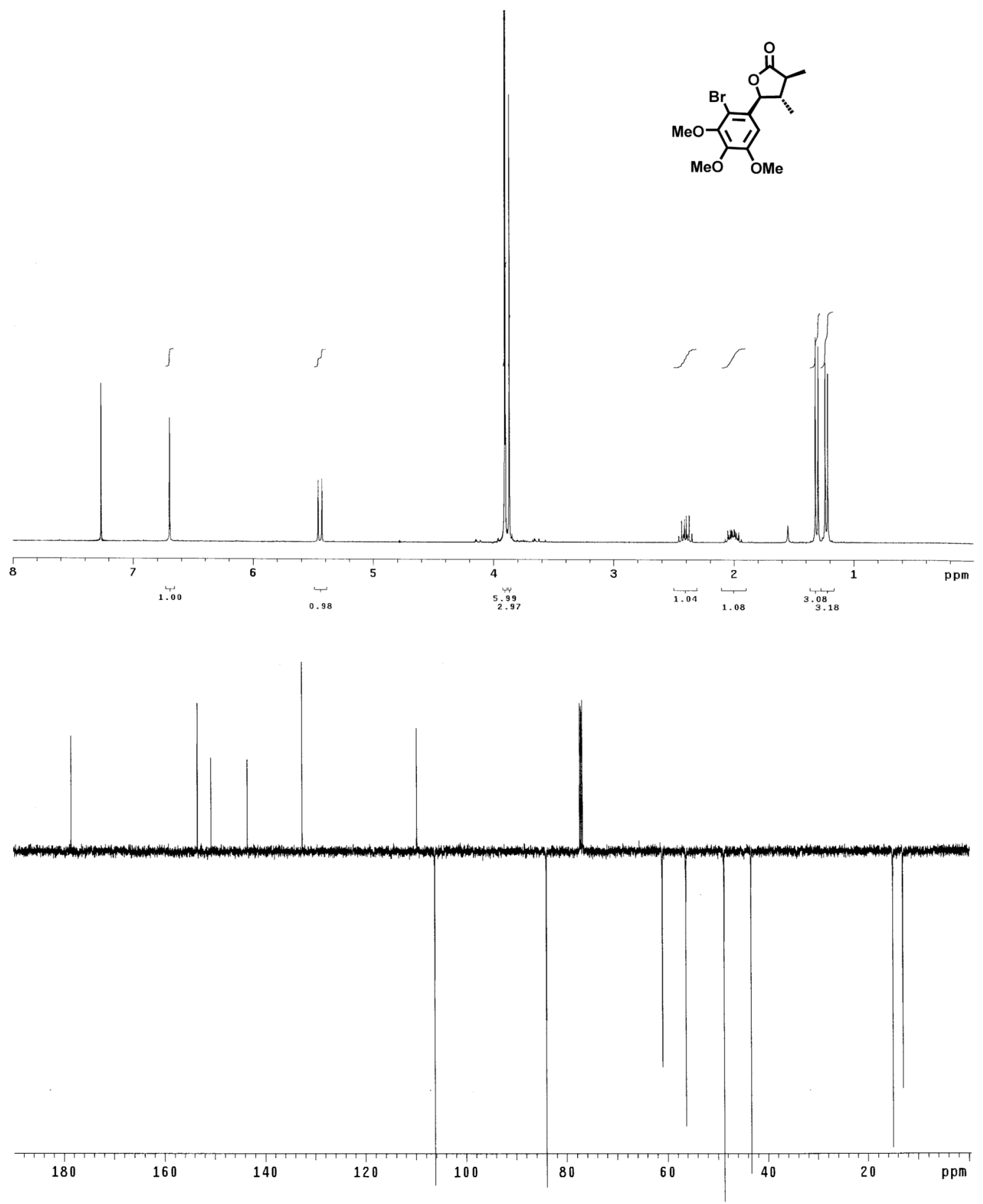
$\left(3 \mathrm{R}^{*}, 4 \mathrm{~S}^{*}, 5 \mathrm{~S}^{*}\right)$-5-(2-Bromo-3,4,5-trimethoxyphenyl)-3,4-dimethyl-dihydro-furan-2-one (10) ${ }^{1} \mathrm{H}(300 \mathrm{MHz})$ and ${ }^{13} \mathrm{C}(125 \mathrm{MHz}) \mathrm{NMR}$ in $\mathrm{CDCl}_{3}$
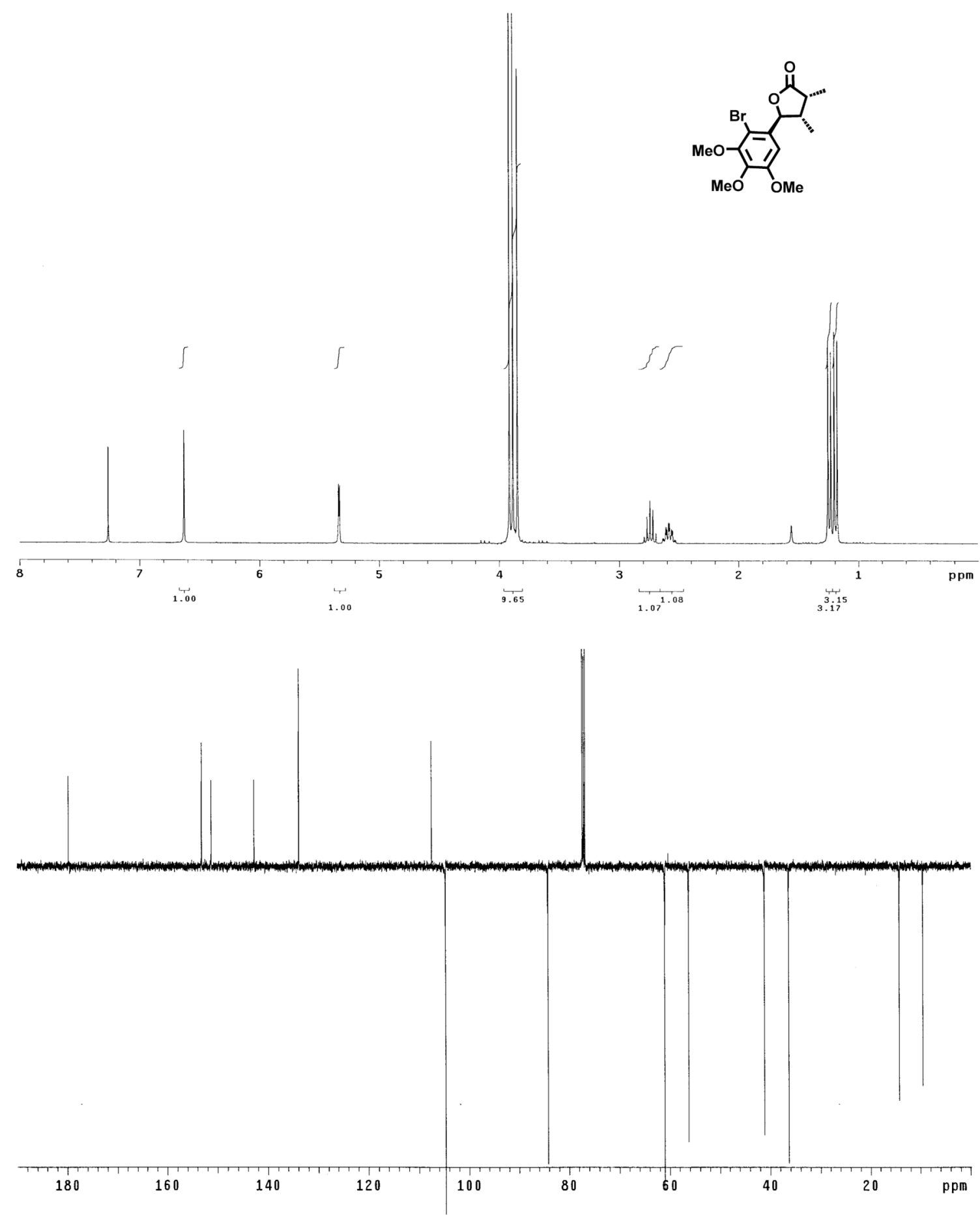
$\left(3 \mathrm{~S}^{*}, 4 \mathrm{R}^{*}, 5 \mathrm{~S}^{*}\right)$-5-(2-Bromo-3,4,5-trimethoxyphenyl)-3,4-dimethyl-dihydro-furan-2-one (14) ${ }^{1} \mathrm{H}(300 \mathrm{MHz})$ and ${ }^{13} \mathrm{C}(125 \mathrm{MHz}) \mathrm{NMR}$ in $\mathrm{CDCl}_{3}$
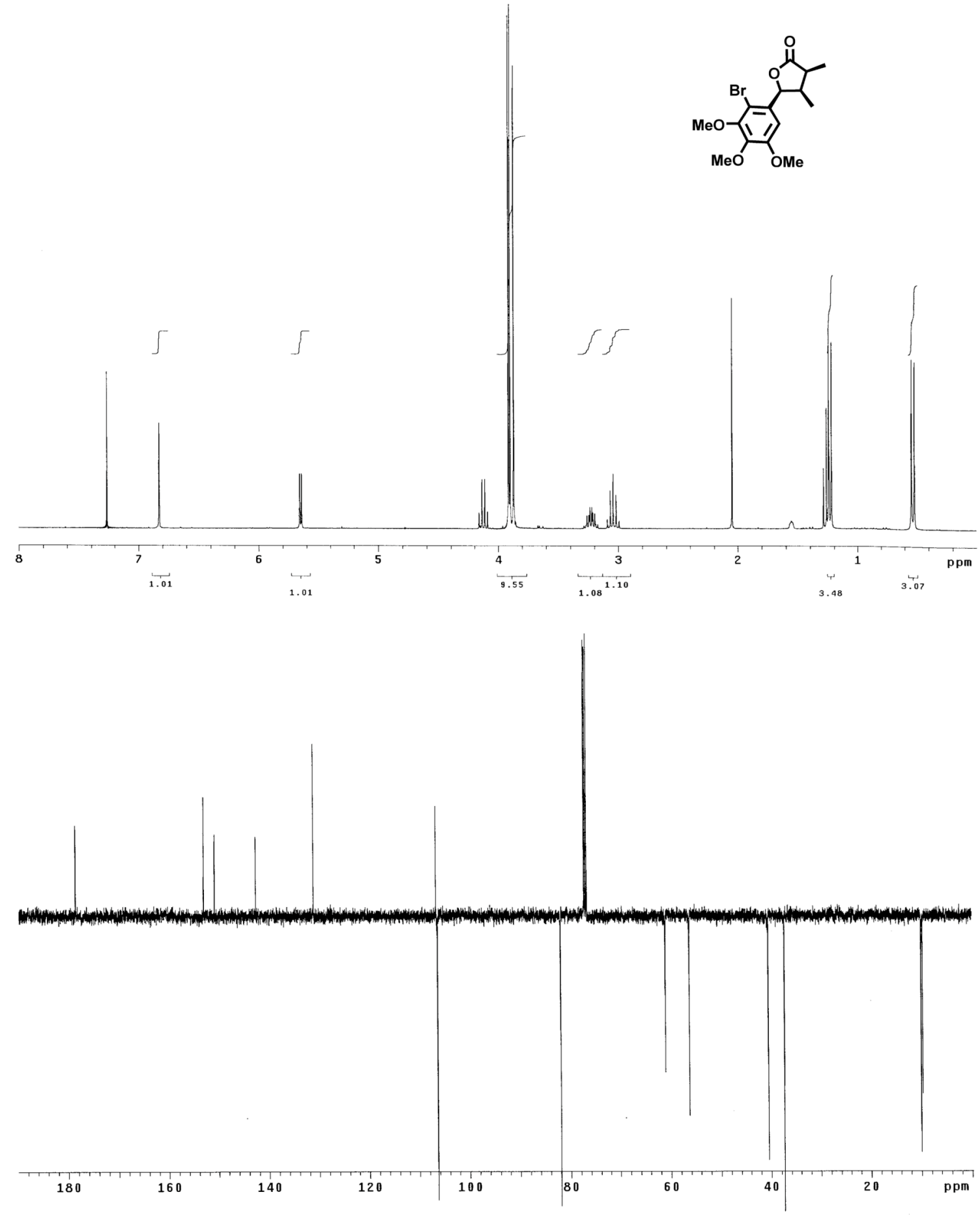
3,4-epi-1

${ }^{1} \mathrm{H}(500 \mathrm{MHz})$ and ${ }^{13} \mathrm{C}(125 \mathrm{MHz}) \mathrm{NMR}$ in $\mathrm{CDCl}_{3}$
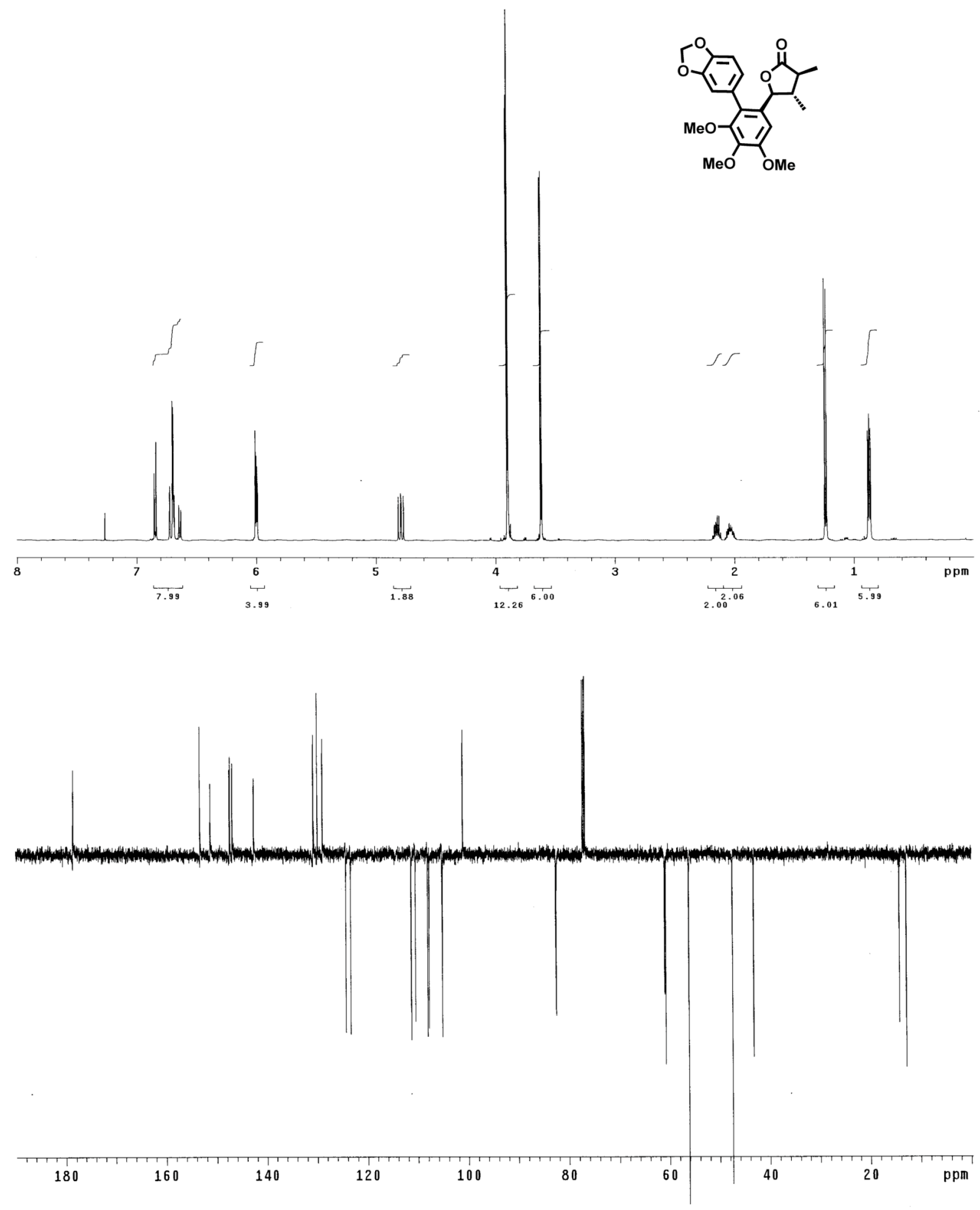
4-epi-1

${ }^{1} \mathrm{H}(400 \mathrm{MHz})$ and ${ }^{13} \mathrm{C}(125 \mathrm{MHz}) \mathrm{NMR}$ in $\mathrm{CDCl}_{3}$
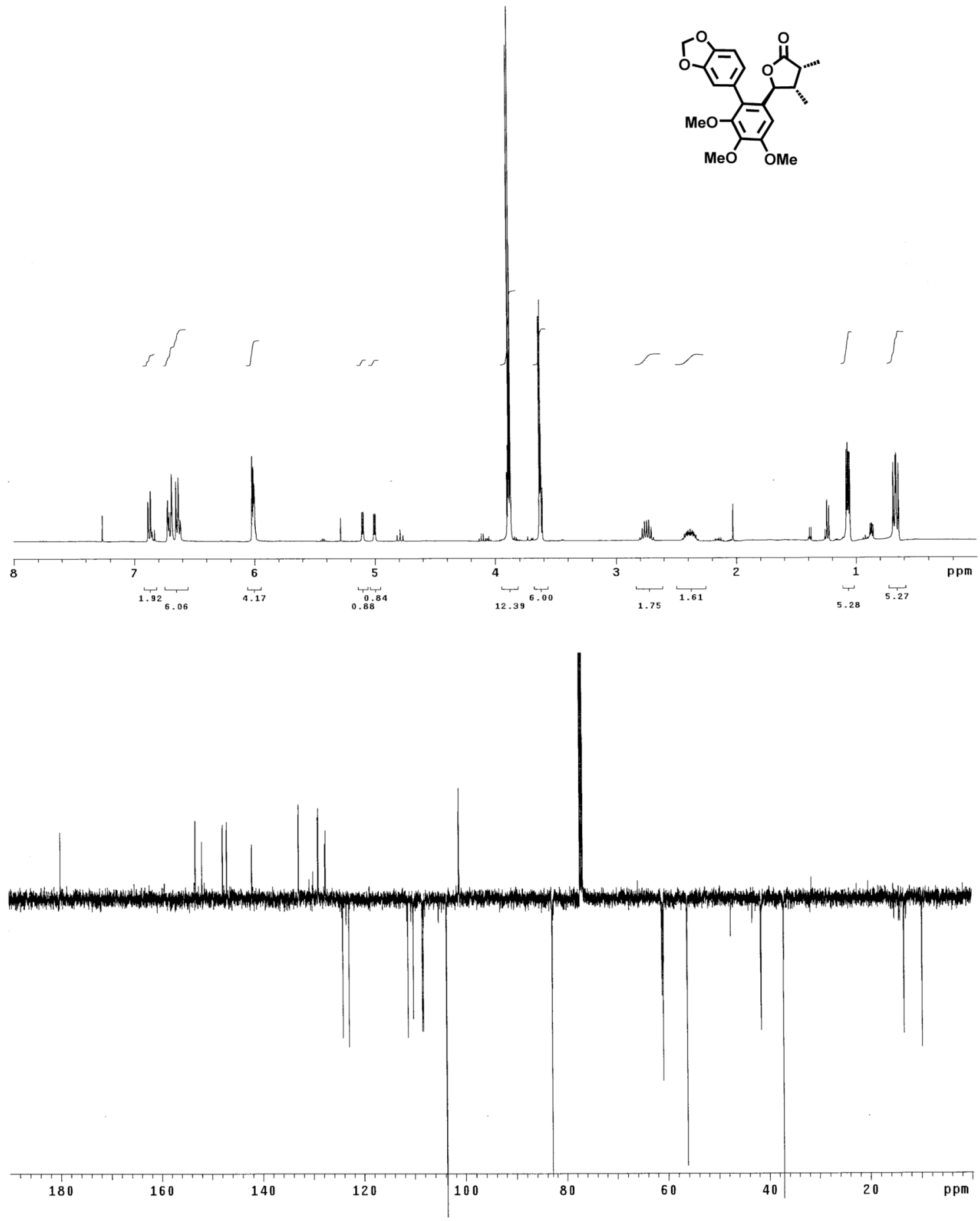
3-epi-1

${ }^{1} \mathrm{H}(300 \mathrm{MHz})$ and ${ }^{13} \mathrm{C}(125 \mathrm{MHz}) \mathrm{NMR}$ in $\mathrm{CDCl}_{3}$
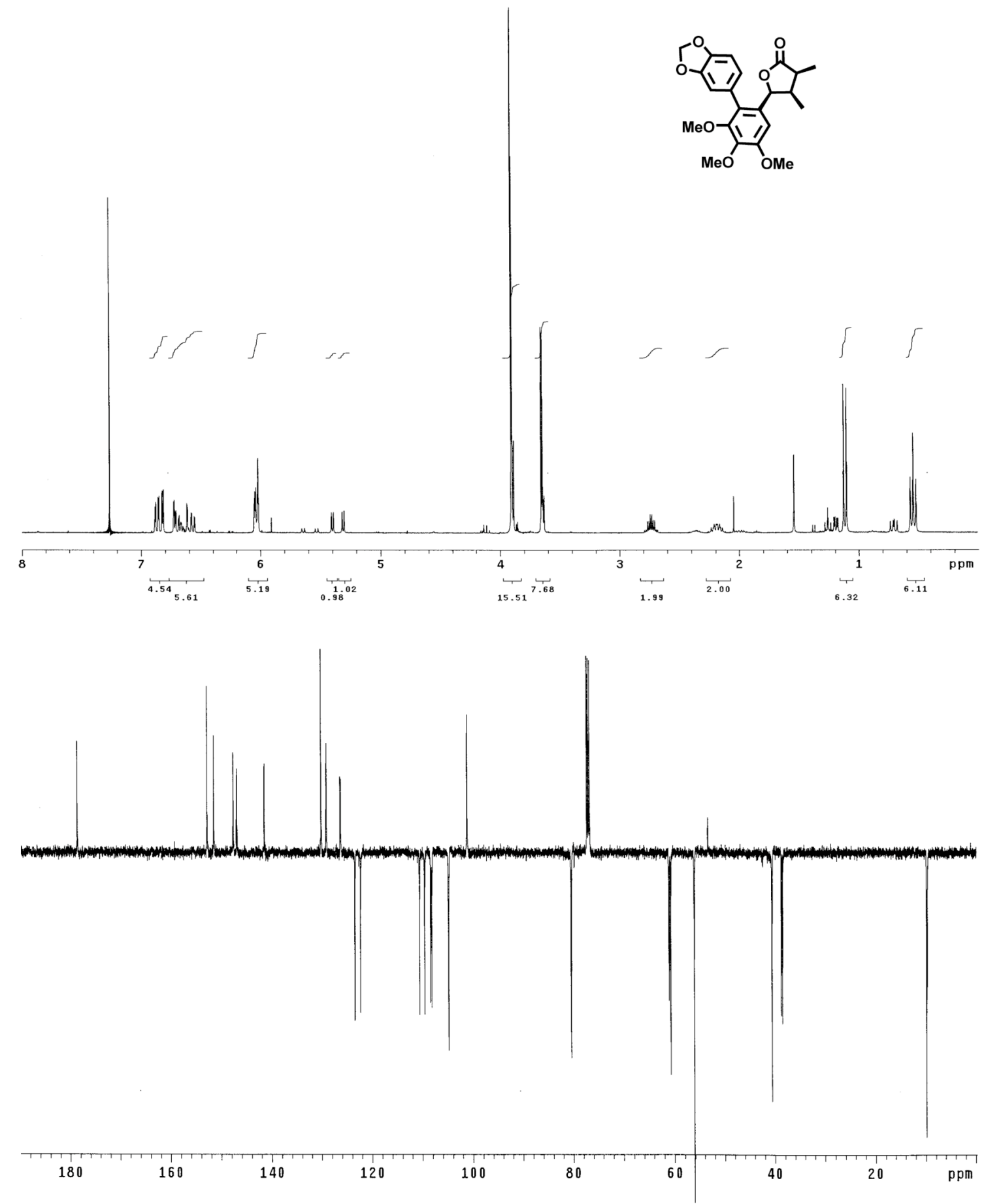
Eupomatilone-6 (1)

${ }^{1} \mathrm{H}(500 \mathrm{MHz}) \mathrm{NMR}$ in $\mathrm{CDCl}_{3}$
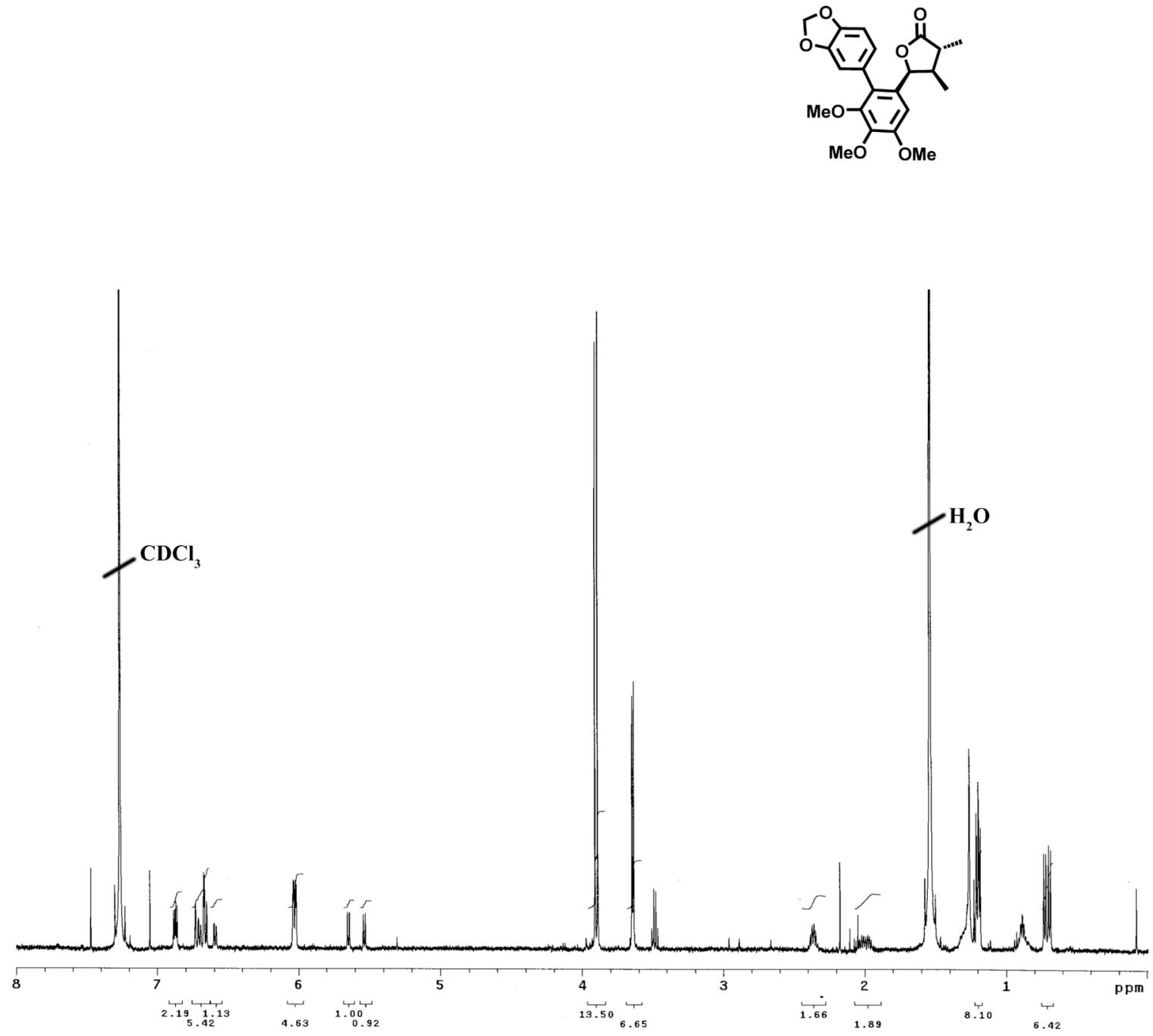
Ethyl (2Z)-2-[(4,4,5,5-tetramethyl-1,3,2-dioxaborolan-2-yl)methyl] but-2-enoate ( $Z$-4)

${ }^{1} \mathrm{H}(300 \mathrm{MHz})$ and ${ }^{13} \mathrm{C}(125 \mathrm{MHz}) \mathrm{NMR}$ in $\mathrm{CDCl}_{3}$
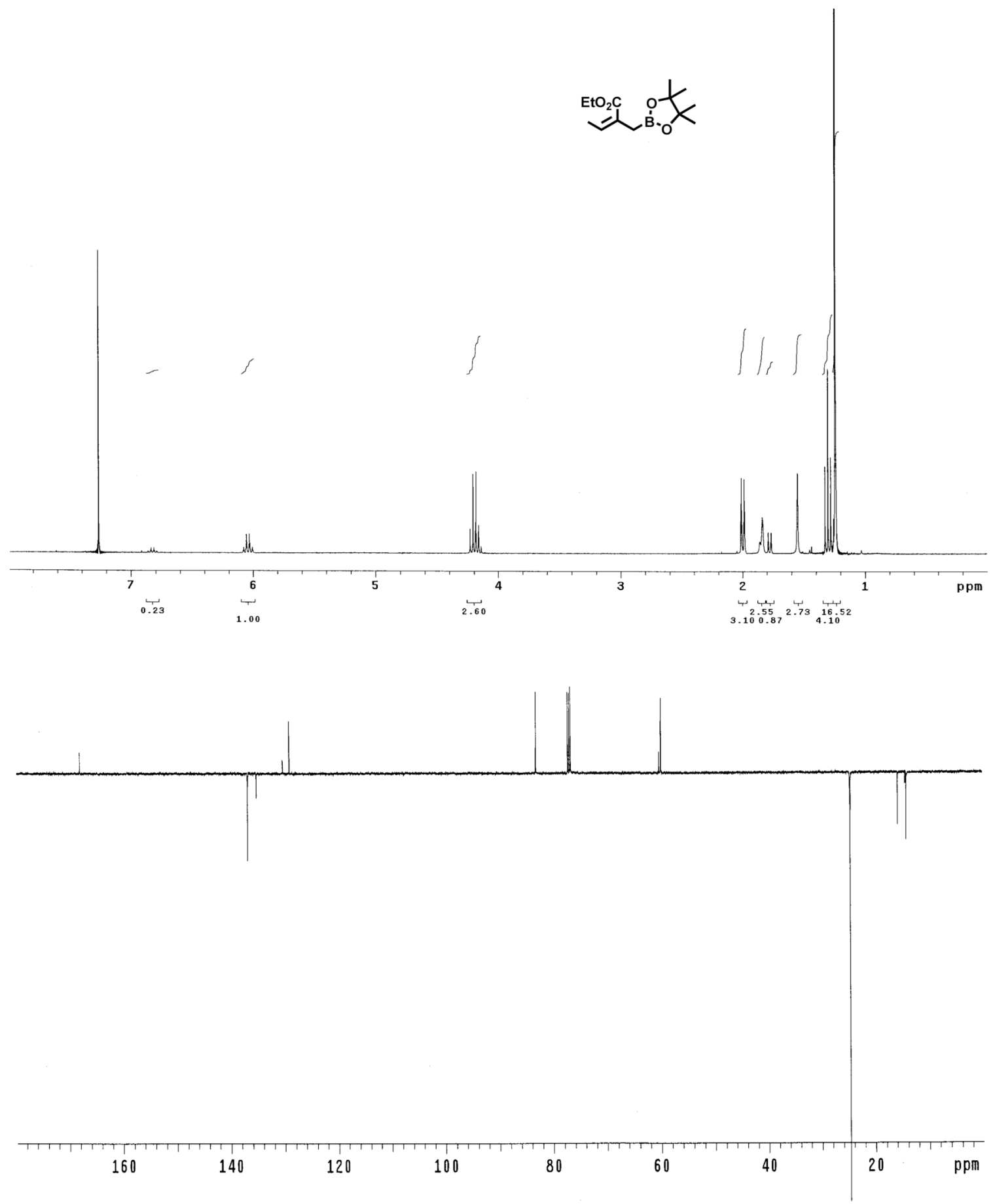
10. Summary table of ${ }^{1} \mathrm{H}$ NMR correspondences for the lactone moiety ${ }^{6,7,11,12}$

\begin{tabular}{|c|c|c|c|c|c|c|c|c|}
\hline & & & \\
\hline
\end{tabular}

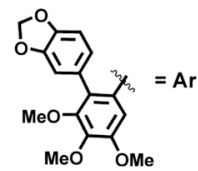


11. ORTEP reproductions for $8,10,14,3,4-$ epi-1, 4-epi-1

rac-(3S*, 4S*, 5S*)-5-(2-Bromo-3,4,5-trimethoxyphenyl)-3,4-dimethyl-dihydrofuran-2-one (8)
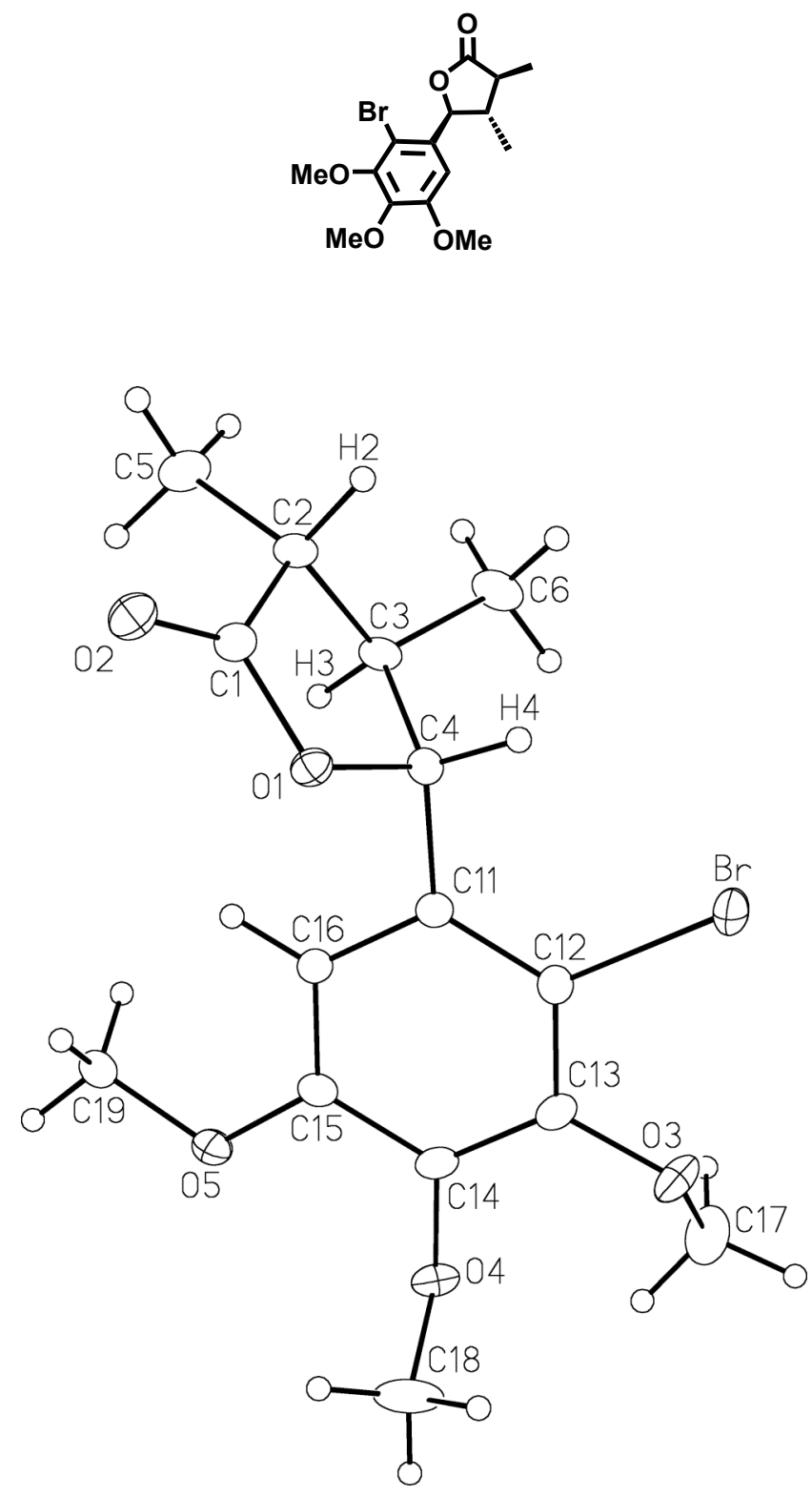
rac-(3R*, 4S*, 5S*)-5-(2-Bromo-3,4,5-trimethoxyphenyl)-3,4-dimethyl-dihydrofuran-2-one (10)
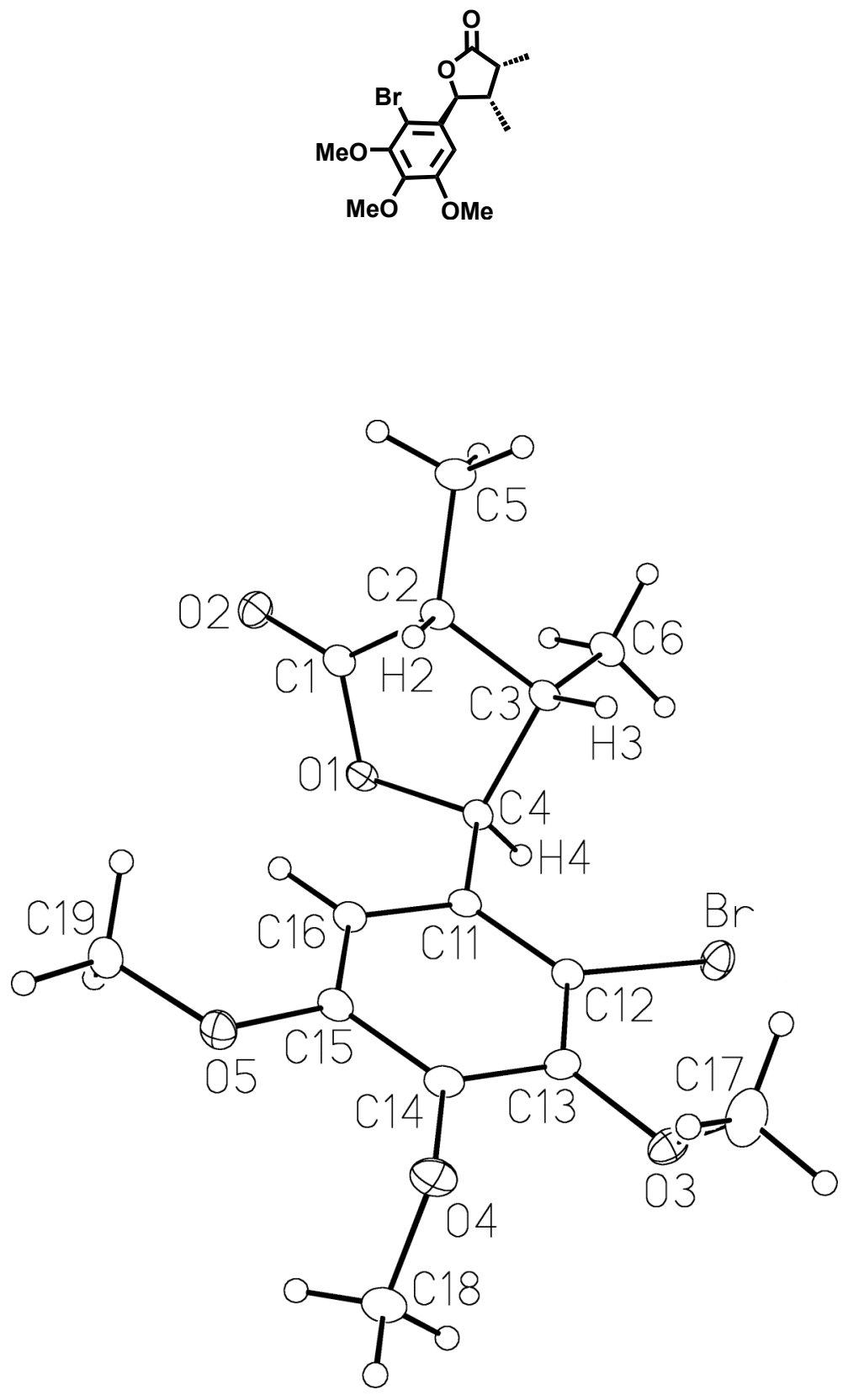
rac-(3S*, 4R*, 5S*)-5-(2-Bromo-3,4,5-trimethoxyphenyl)-3,4-dimethyl-dihydrofuran-2-one (14)
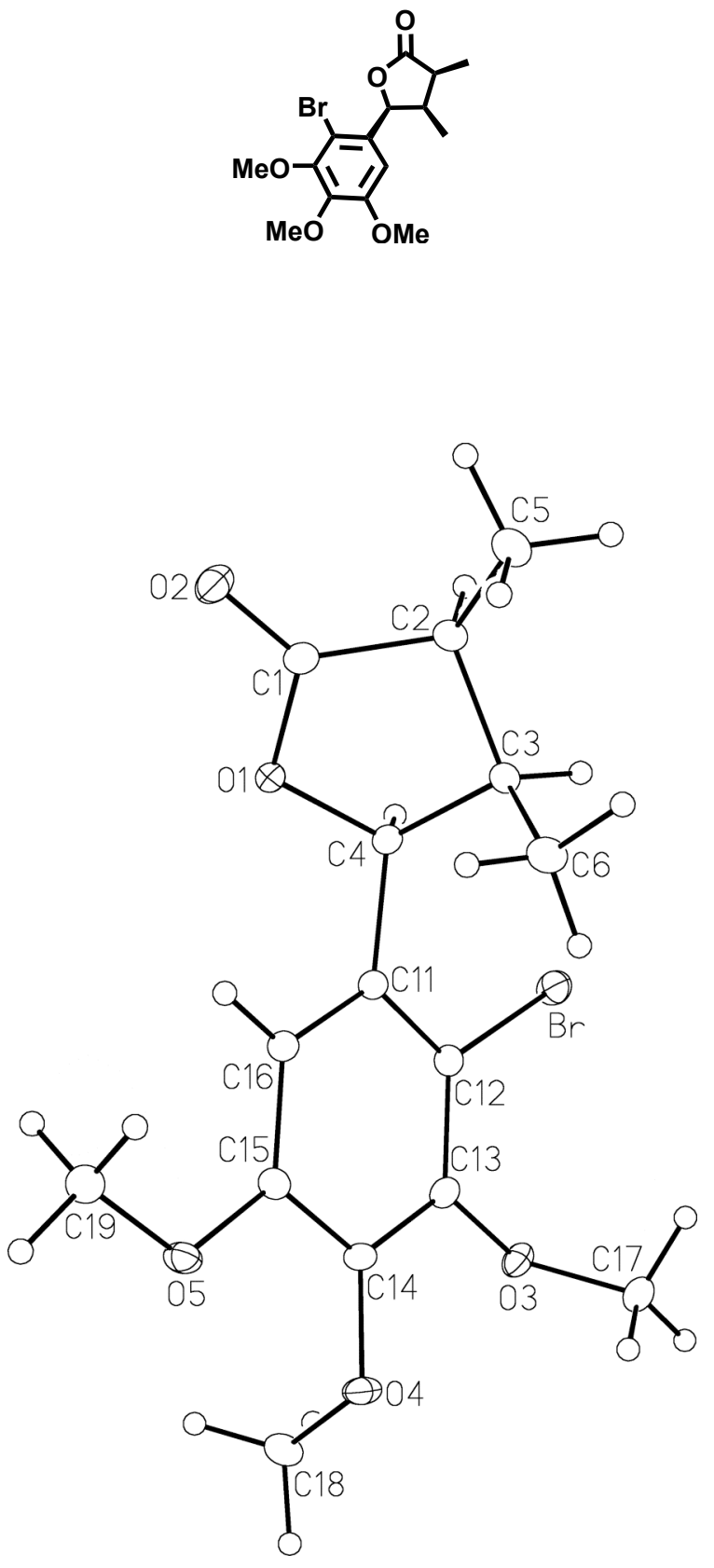


\section{3,4-epi-1}
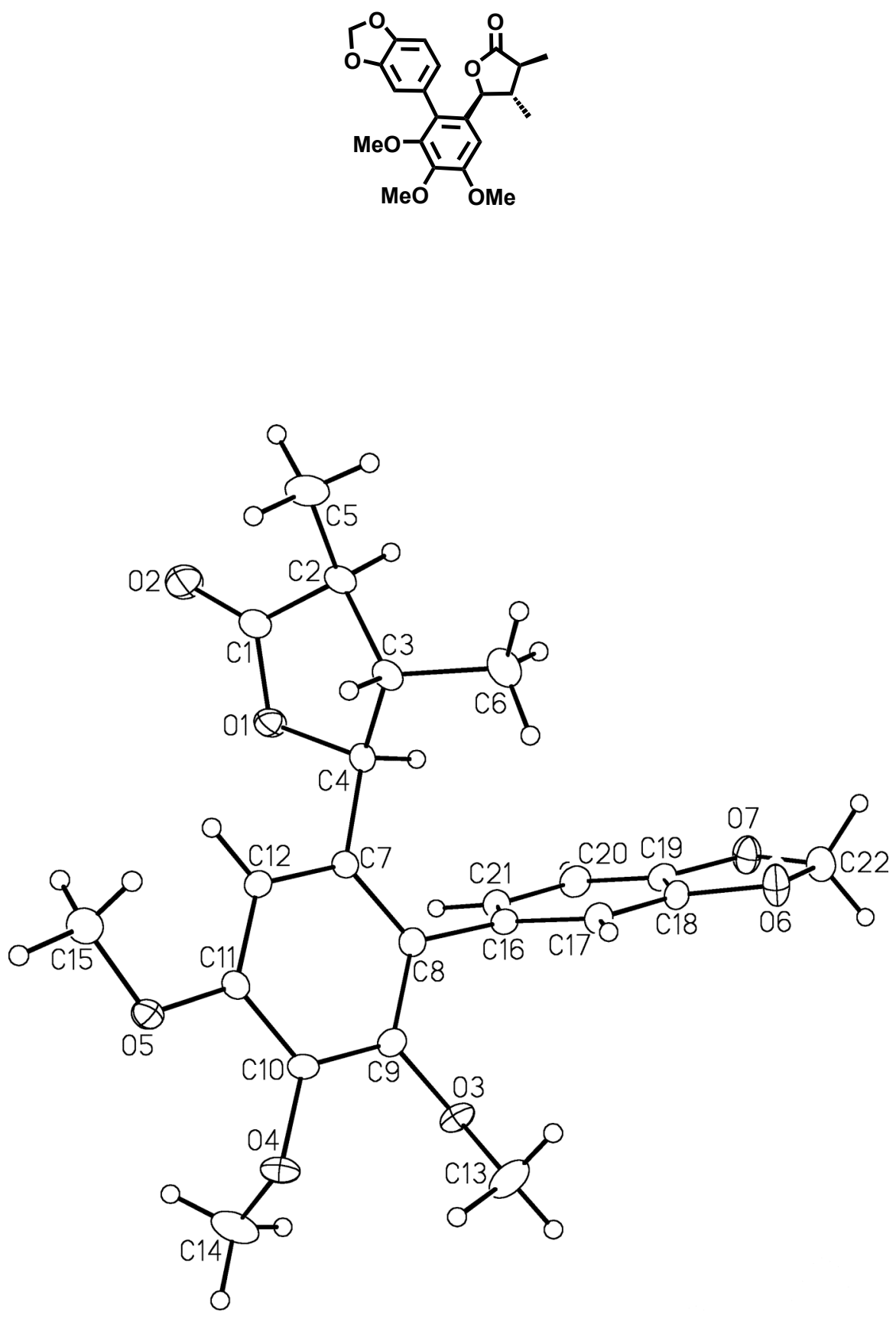


\section{4-epi-1}
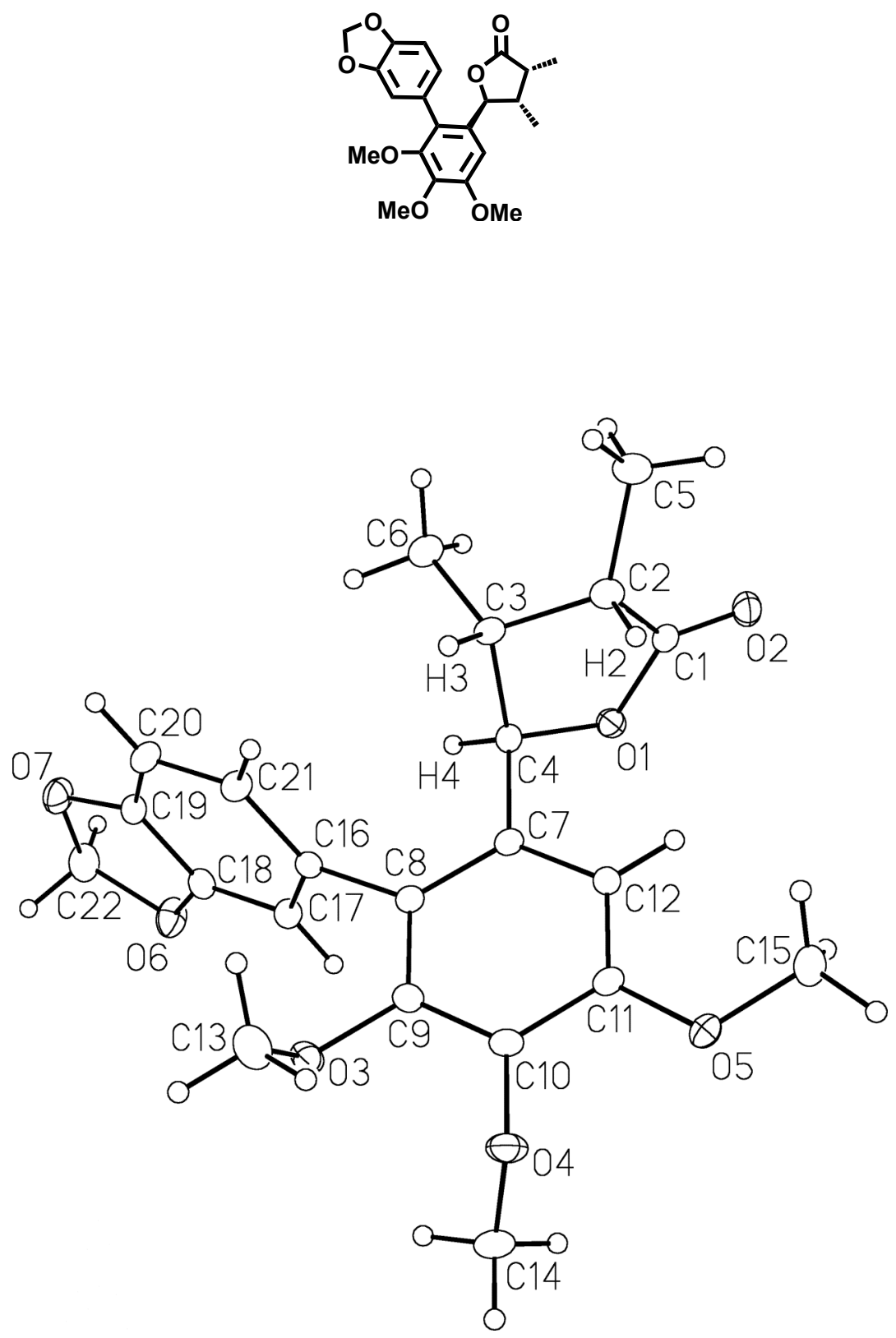


\section{References}

1. Taylor, R. K.; Casy, G. In Organocopper Reagents: A Practical Approach;

Taylor, R. K., Ed.; Oxford University Press: New York, 1994, pp 53-72.

2. Phillion, D. P.; Neubauer, R.; Andrew, S. S. J. Org. Chem. 1986, 51, 1610-1612.

3. Molander, G. A.; George, K. M.; Monovich, L. G. J. Org. Chem. 2003, 68, 95339540.

4. Kennedy, J. W. J.; Hall, D. G. J. Org. Chem. 2004, 69, 4412-4428.

5. Barder, T. E.; Walker, S. D.; Martinelli, J. R.; Buchwald, S. L. J. Am. Chem. Soc. 2005, 127, 4685-4696.

6. Hutchison, J. M.; Hong, S.-P.; McIntosh, M. C. J. Org. Chem. 2004, 69, 41854191.

7. Coleman, R. S.; Gurrala, S. R. Org. Lett. 2004, 6, 4025-4028.

8. Pinto, A. C.; Freitas, C. B. L.; Dias, A. G.; Pereira, V. L. P.; Tinant, B.; Declercq, J.-P.; Costa, P. R. R. Tetrahedron: Asymmetry 2002, 13, 1025-1031.

9. a) Ramachandran, P. V.; Pratihar, D.; Biswas, D.; Srivastava, A.; Reddy, M. V. R. Org. Lett. 2004, 6, 481-484. b) Nyzam, V.; Belaud, C.; Villiéras, J. Tetrahedron Lett. 1993, 34, 6899-6902.

10. Whiting, A. Tetrahedron Lett. 1991, 32, 1503-1506.

11. Carroll, A. R.; Taylor, W. C. Aust. J. Chem. 1991, 44, 1705-1714.

12. Gurjar, M. K.; Cherian, J.; Ramana, C. V. Org. Lett. 2004, 6, 317-319. 